COMMUNICATIONS IN

ANALYSIS AND GEOMETRY

Volume 7, Number 3, 641-679, 1999

\title{
Spinors and forms on the ball and the generalized cone
}

\author{
J.S.DOWKeR AND Klaus Kirsten
}

\begin{abstract}
A method is presented, and used, for determining any heat-kernel coefficient for the form-valued Laplacian on the $D$-ball as an explicit function of dimension and form order. The calculation is offerred as a particular application of a general technique developed earlier for obtaining heat-kernel coefficients on a bounded generalized cone which involves writing the sphere and ball $\zeta$-functions, and coefficients, in terms of Barnes $\zeta$-functions and generalized Bernoulli polynomials. Functional determinants are computed. Spinors are also treated by the general method.
\end{abstract}

\section{Introduction.}

The present work should be considered as an extension of an earlier one, [8] where heat-kernel asymptotics and functional determinants were computed for a scalar field on a bounded generalized cone (the simplest example of which is a ball). Here, the scalar field is replaced by more complicated bundles, specifically by spinors and forms. This will enable us later to discuss topological matters such as the analytic torsion as well as settling a few questions regarding the spectral invariants. However the emphasis here will be on the actual computations and the technical problems raised have, we think, elegant outcomes. A primary aim is to work in arbitrary dimensions with arbitrary $p$-forms. In this connection, one objective is to make contact with the work of Blažić et al [5] who have given general, explicit expressions for the first four heat-kernel coefficients for the form-valued Laplacian on manifolds with boundary. We anticipate going beyond their computations but, of course, within the confines of a restricted geometry.

The motivation for this calculation is partly mathematical and partly physical. It fits into that part of mathematics termed spectral geometry, and adds, we hope, to the to general stock of specifics in that area. In physics, heat-kernel expansions and functional determinants have been heavily employed in quantum field theory, especially in quantum cosmology. 
In the progress of the analysis, we develop various ancillary results and techniques that we hope will be of value to others who wish to undertake similarly detailed calculations. Although the bulk of this paper is concerned with the ball, there is no obstruction to discussing conical cases in like detail. The results would then be relevant to the investigation of the effects of singularities and of the non-smoothness of the boundary on the spectral invariants.

The structure of this paper is as follows. In section 2, we discuss the various types of eigenforms that exist on the generalized cone. For rapidity, we use the results of Cheeger [14] on the infinite cone, additionally imposing appropriate conditions at the cone boundary.

Section 3 contains the general construction of the $\zeta$-function and heatkernel coefficients. This closely follows our earlier development so that explanatory details can be kept to a minimum. The result is a formula for the $p$-form coefficients on the generalized cone in terms of those on its base for a particular, 'modified' coexact $p$-form.

In sections 4 and 5 this apparatus is applied to the case of the ball where it is possible to find a compact expression for the relevant $p$-form $\zeta$-function on the sphere in terms of Barnes $\zeta$-functions. Another more suggestive form of this sphere $\zeta$-function expresses it as a sum of 0 -form $\zeta$-functions. The ball coefficients are determined as explicit functions of $d$ and $p$ via generalized Bernoulli functions.

Rearranging the first few coefficients we obtain agreement with the general formulae of [5] evaluated on the ball. Going beyond this, we are able, in section 6 , to find the expression for an arbitrary coefficient in terms of binomial coefficients and polynomials in the dimension. This is a natural generalisation of our previous results [8], and of Levitin's earlier [31], for the scalar case. Section 7 briefly looks at the important value of the $\zeta$-function evaluated at zero argument.

In section 8 we address the calculation of the functional determinants and some explicit results are exhibited in an appendix.

Related work has been performed by Elizalde et al [21] and we use their specific results as a check of our general forms. Their method of evaluation also works generally but involves an immediate reduction to a series of Hurwitz $\zeta$-functions and does not yield the functional form. The expressions for the functional determinants obtained in [21] contain integrals over $\Psi$ and $\Gamma$ functions. In an appendix we show how these may be reduced to derivatives of the Riemann $\zeta$-function.

In section 9 we turn, rather more briefly, to spinors and apply our general method to rapidly reproduce and justify our earlier results for spectral 
boundary conditions. A curious vanishing theorem, noticed earlier, is proved in an appendix. Local conditions are also treated by the improved technique.

\section{Geometry and eigenforms.}

The geometry has been outlined in Cheeger [14] and already used in our earlier work, [8], so that it will not be described at length here. The cone manifold, $\mathcal{M}$ has the hyperspherical polar metric $d s^{2}=d r^{2}+r^{2} d \Sigma^{2}$ with $d \Sigma^{2}$ the metric on the base, $\mathcal{N}$, which is the $r=1$ section of the cone. For simplicity, we shall assume in this paper that the base is closed, $\partial \mathcal{N}=\emptyset$, which is enough for the ball.

The structure of the eigenforms of the de Rham Laplacian, $\Delta_{\mathcal{M}}$, on the infinite generalized cone has been given by Cheeger [14] and, for convenience, we refer to [14] for the detailed action of $\Delta_{\mathcal{M}}$ on forms of the type $\beta+d r \wedge \omega$ as well as for the necessary exterior calculus. (See also [15].)

There are four basic types of eigenforms with nonzero eigenvalues, $\alpha^{2}$,

$$
\begin{aligned}
\phi_{p}^{\mathcal{M}(1)} & =\frac{J_{\nu(p)}(\alpha r)}{r^{(d-1-2 p) / 2}} \phi_{p}^{\mathcal{N}} \\
\phi_{p}^{\mathcal{M}(2)} & =\frac{J_{\nu(p-1)}(\alpha r)}{r^{(d+1-2 p) / 2}} \tilde{d}_{p-1}^{\mathcal{N}}+\left(\frac{J_{\nu(p-1)}(\alpha r)}{r^{(d+1-2 p) / 2}}\right)^{\prime} d r \wedge \phi_{p-1}^{\mathcal{N}}, \\
\phi_{p}^{\mathcal{M}(3)} & =\frac{1}{r^{d-2 p}}\left(r^{(d+1-2 p) / 2} J_{\nu(p-1)}(\alpha r)\right)^{\prime} \tilde{d} \phi_{p-1}^{\mathcal{N}} \\
& +\frac{J_{\nu(p-1)}(\alpha r)}{r^{(d+3-2 p) / 2}} d r \wedge \tilde{\delta} \tilde{d} \phi_{p-1}^{\mathcal{N}}, \\
\phi_{p}^{\mathcal{M}(4)} & =\frac{J_{\nu(p-2)}(\alpha r)}{r^{(d+1-2 p) / 2}} d r \wedge \tilde{d} \phi_{p-2}^{\mathcal{N}},
\end{aligned}
$$

where $\phi_{p}^{\mathcal{N}}$ is a coexact $p$-eigenform of the intrinsic de Rham Laplacian, $-\Delta_{\mathcal{N}}=\tilde{d} \tilde{\delta}+\tilde{\delta} \tilde{d}$, on the base $\mathcal{N}$. We have the separation of variables relation

$$
\nu(p)=\left(\mu(p)+((d-1) / 2-p)^{2}\right)^{1 / 2}
$$

with $\mu(p)$ being the (coexact) eigenvalue of $\Delta_{\mathcal{N}}$. We are assuming that $\nu \geq 1$ so that the 'negative' modes $\sim J_{-\nu}$ do not arise.

In addition, there are modes ('zero modes') whose $\mathcal{N}$ part is harmonic, $\mu=0$,

$$
\phi_{p}^{\mathcal{M}(E)}=\frac{J_{\nu_{E}(p)}(\alpha r)}{r^{(d-1-2 p) / 2}} h_{p}^{\mathcal{N}}
$$


and

$$
\phi_{p}^{\mathcal{M}(O)}=\left(\frac{J_{\nu_{O}(p)}(\alpha r)}{r^{(d+1-2 p) / 2}}\right)^{\prime} d r \wedge h_{p-1}^{\mathcal{N}}
$$

with

$$
\begin{aligned}
& \nu_{E}(p)=|(d-1) / 2-p|=\nu_{E}(d-1-p) \\
& \nu_{O}(p)=|(d+1) / 2-p|=\nu_{E}(d-p) .
\end{aligned}
$$

On $\mathcal{M}$, types $1, E$ and 3 are coexact and types $2, O$ and 4 are exact. (See the relations in [15].)

For the bounded, generalized cone, conditions are to be set at $r=1$. Absolute boundary conditions are $[16,26]$

$$
\left.\left(\phi_{i \ldots j}^{\mathcal{M}}\right)^{\prime}\right|_{\mathcal{N}}=0,\left.\quad \phi_{r, i \ldots j}^{\mathcal{M}}\right|_{\mathcal{N}}=0
$$

and have to be applied to the six types separately.

Since $\phi_{p}^{\mathcal{N}}, h_{p}^{\mathcal{N}}$ are pure $\mathcal{N}$ forms, it is easily shown that types $1,2, E$ and $O$ satisfy Neumann (Robin) and types 3 and 4 Dirichlet conditions. Bessel's equation has to be used to derive this for type 3 .

More precisely the Robin conditions are (e.g. for type 1 )

$$
\left.\partial_{r}\left(r^{p-(d-1) / 2} J_{\nu(p)}(\alpha r)\right)\right|_{\mathcal{N}}=0
$$

so that the Robin parameter is $u=u_{a}(p)=p-(d-1) / 2$, ([7] 3.1). The parameter for type 2 is $u_{a}(p-1)$.

Relative boundary conditions are obtained by dualising and, in the present context amount to

$$
\left.\left(\partial_{r}+d+2-2 p\right) \phi_{r, i \ldots j}^{\mathcal{M}}\right|_{\mathcal{N}}=0,\left.\quad \phi_{i \ldots j}^{\mathcal{M}}\right|_{\mathcal{N}}=0
$$

Now types 1, 2, $E$ and $O$ satisfy Dirichlet and types 3 and 4 Robin conditions. For type 3, Robin conditions read

$$
\left.\partial_{r}\left(r^{(d+1) / 2-p} J_{\nu(p-1)}(\alpha r)\right)\right|_{\mathcal{N}}=0
$$

with the Robin parameter $u=u_{r}(p)=(d+1) / 2-p=u_{a}(d-p)$. The parameter for type 4 is $u_{r}(p-1)$. Hodge $\star$-duality on $\mathcal{M}$ thus interchanges 
the conditions (10) and (12) with $1 \leftrightarrow 4$ and $2 \leftrightarrow 3$ which differs from Cheeger.

We denote the coexact degeneracies on $\mathcal{N}$ by $d(p)$ and remark that the exact degeneracies, $d_{e x}(p)$, and eigenvalues, $\mu_{e x}(p)$, are given by

$$
d_{e x}(p)=d(p-1), \quad \mu_{e x}(p)=\mu(p-1) .
$$

The structure of the eigenforms shows that the degeneracies of types 1, 2, 3 and 4 are $d(p), d(p-1), d(p-1)$ and $d(p-2)$ respectively.

We now define, for later use, the modified coexact $\zeta$-function on $\mathcal{N}$, the base $\zeta$-function,

$$
\zeta_{p}^{\mathcal{N}}(s)=\sum \frac{d(p)}{\nu(p)^{2 s}}=\sum \frac{d(p)}{\left(\mu(p)+((d-1) / 2-p)^{2}\right)^{s}}
$$

(cf [8] (3.3)). Including the 'zero modes', which is sometimes notationally convenient, one has

$$
\tilde{\zeta}_{p}^{\mathcal{N}}(s)=\zeta_{p}^{\mathcal{N}}(s)+\frac{\beta_{p}^{\mathcal{N}}}{\nu_{E}(p)^{2 s}} \equiv \sum \frac{\tilde{d}(p)}{\nu(p)^{2 s}}
$$

which defines $\tilde{d}(p)$ and where $\beta_{p}^{\mathcal{N}}$ is the $p$-th Betti number of $\mathcal{N}$. The summations, here and later, are over the mode labels which are not always explicitly displayed.

In view of the relations (13), an 'exact' $\zeta$-function is defined by

$$
\zeta_{e x, p}^{\mathcal{N}}(s)=\zeta_{p-1}^{\mathcal{N}}(s) .
$$

Because the additional term $((d-1) / 2-p)^{2}$ depends on $p, \zeta_{e x, p}^{\mathcal{N}}(s)$ is not the true exact $\zeta$-function, but we expect it to be the relevant construct.

Assume for the time being that the base $\mathcal{N}$ has no boundary. Hodge duality on $\mathcal{N}$ can be applied to yield the coexact relations

$$
d(d-1-p)=d(p), \quad \text { and } \quad \mu(d-1-p)=\mu(p)
$$

whence

$$
\nu(d-1-p)=\nu(p)
$$

and therefore, for the coexact $\zeta$-function,

$$
\zeta_{d-1-p}^{\mathcal{N}}(s)=\zeta_{p}^{\mathcal{N}}(s)
$$


These relations will be verified later when $\mathcal{N}$ is a sphere.

As is well known, equation (13) is true for any Riemannian manifold. This follows from the isomorphism between the nonzero exact and coexact eigenspaces provided by the exterior derivative. It allows alternating sums over $p$ to be simplified by 'téléscopage' [27]. (See e.g. the early use by Ray in analytic torsion [35]).

The binomial equivalent is the identity

$$
\left(\begin{array}{c}
D-1-r \\
p-m
\end{array}\right)=\sum_{q=0}^{p}(-1)^{p-q}\left(\begin{array}{c}
D-r \\
q-m
\end{array}\right) .
$$

\section{General constructions.}

The total $\zeta$-function on $\mathcal{M}$ is, according to (1) - (4), (6), (7), a combination of exact and coexact (on $\mathcal{M}$ ) contributions. Noting that the zero modes on $\mathcal{N}$ give degenerate eigenvalues on $\mathcal{M}$, one has

$$
\zeta_{p}^{\mathcal{M}+}(s)=\sum_{i=1}^{4} \sum_{\alpha_{i}} \frac{1}{\alpha_{i}^{2 s}}+\beta_{p}^{\mathcal{N}} \sum_{\alpha_{E}} \frac{1}{\alpha_{E}^{2 s}}+\beta_{p-1}^{\mathcal{N}} \sum_{\alpha_{O}} \frac{1}{\alpha_{O}^{2 s}}
$$

where the $\alpha_{i}^{2 s}$ are the eigenvalues of the $p$-form Laplacian on $\mathcal{M}$ for $i$-type modes.

(21) can be written in terms of the coexact $\zeta$-function on $\mathcal{M}, \zeta_{p}^{\mathcal{M}}(s)$, as

$$
\zeta_{p}^{\mathcal{M}+}(s)=\zeta_{p}^{\mathcal{M}}(s)+\zeta_{p-1}^{\mathcal{M}}(s)
$$

the inverse of which is

$$
\zeta_{p}^{\mathcal{M}}(s)=\sum_{q=0}^{p}(-1)^{p-q} \zeta_{q}^{\mathcal{M}+}(s) .
$$

Equation (22) holds for any manifold, i.e. the total $\zeta$-function is always the sum of coexact $\zeta$-functions and (23) is just a consequence of téléscopage.

According to the method given in $[6,7,8]$, and now for absolute conditions,

$$
\begin{aligned}
\zeta_{a, p}^{\mathcal{M}}(s)=\sum \int_{\gamma} \frac{d k}{2 \pi i} k^{-2 s} \frac{\partial}{\partial k}\left(\left.\tilde{d}(p) \ln \left(r^{u_{a}(p)} J_{\nu(p)}(k r)\right)^{\prime}\right|_{\mathcal{N}}\right. \\
\left.+d(p-1) \ln J_{\nu(p-1)}(k)\right)
\end{aligned}
$$


where the details of the contour $\gamma$ are in the above cited references. The first term is the Neumann (Robin) (types 1 and $E$ ) and the second term the Dirichlet (type 3) part. For economy of writing, the degeneracy $\tilde{d}(p)$ is introduced so as to take into account the $\beta_{p}^{\mathcal{N}} E$-type zero modes on $\mathcal{N}, c f$ (15). When $p \rightarrow p-1$, the type 1 contribution becomes a type 2 , the type $E$ a type $O$ and the type 3 a type 4 .

For relative conditions, likewise,

$$
\begin{aligned}
\zeta_{r, p}^{\mathcal{M}}(s)=\sum \int_{\gamma} \frac{d k}{2 \pi i} k^{-2 s} \frac{\partial}{\partial k}\left(\left.d(p-1) \ln \left(r^{u_{r}(p)} J_{\nu(p-1)}(k r)\right)^{\prime}\right|_{\mathcal{N}}\right. \\
\left.+\tilde{d}(p) \ln J_{\nu(p)}(k)\right) .
\end{aligned}
$$

It is amusing to check in detail Hodge duality on $\mathcal{M}$,

$$
\zeta_{a, p}^{\mathcal{M}+}(s)=\zeta_{r, d+1-p}^{\mathcal{M}+}(s),
$$

which fundamentally arises from the intertwining [26]

$$
\star \Delta_{p, a}^{\mathcal{M}}=\Delta_{d+1-p, r}^{\mathcal{M}} \star .
$$

It is easily seen from (22) that (26) is equivalent to the statement that, under $p \rightarrow d-p, \zeta_{a, p}^{\mathcal{M}}(s)$ of (24) turns into $\zeta_{r, p}^{\mathcal{M}}(s)$ of (25). Firstly, this is readily seen to be the case from the relations (17) and (18), if the zero $E$ modes are set aside. Secondly, consider the contribution coming from the zero $E$ modes in (24). One has $u_{a}(p)=p-(d-1) / 2$ and the argument of the logarithm reads explicitly $u_{a}(p) J_{\nu_{E}(p)}+k J_{\nu_{E}(p)}^{\prime}$ where $\left|u_{a}(p)\right|=\nu_{E}(p)$. Let us assume for the moment that $u_{a}(p) \leq 0$, and then use the relation $z J_{\nu}^{\prime}(z)-\nu J_{\nu}(z)=-z J_{\nu+1}(z)$ to write the above argument as $-k J_{\nu_{E}(p)+1}$.

The factor $(-k)$ does not contribute because it has no zero inside the contour, $\gamma$, and so the contribution is just that of $J_{\nu_{E}(p)+1}$. Now from (8), $\nu_{E}(p)+1=\nu_{E}(d-p)$ and, taking into account Poincaré duality on $\mathcal{N}, \beta_{p}^{\mathcal{N}}=\beta_{d-p}^{\mathcal{N}}$, the contribution of the $p$-form zero modes for absolute boundary conditions is seen to equal that of the $(d-p)$-form zero modes for relative boundary conditions, which was to be shown in order to complete the demonstration of Hodge duality on $\mathcal{M}$. For $u_{a}(p)>0$ use $z J_{\nu}^{\prime}(z)+\nu J_{\nu}(z)=$ $z J_{\nu-1}(z)$ to arrive at the same conclusion.

One of our main objectives is the evaluation of the coefficients, $A_{n / 2}$, in the heat-kernel expansion, which is stated in generic form,

$$
K^{\mathcal{M}}(\tau)=\sum_{n=0}^{\infty} A_{n / 2}^{\mathcal{M}} \tau^{(n-D) / 2}+A_{\mathcal{M}}^{\prime} \log \tau
$$


Our preferred general computational formula, on any manifold, $\mathcal{M}$, of dimension $D$, is

$$
A_{n / 2}^{\mathcal{M}}=\Gamma((D-n) / 2) \operatorname{Res} \zeta^{\mathcal{M}}((D-n) / 2), \quad n<D .
$$

The ability, by choosing $D$ sufficiently large, to work with just this formula in order to determine any coefficient, has an additional advantage because a finite number of 'extra' modes does not affect the analytic structure, as in the difference between $\zeta$ and $\tilde{\zeta}$ when (28) is applied to the base $\mathcal{N}$.

The calculation of the $\zeta$-function on $\mathcal{M}$ in its relation to that on $\mathcal{N}$, follows precisely the previous pattern, [8], the Robin and Dirichlet cases now being combined. It leads to the following basic equation for the coexact heat-kernel coefficients on $\mathcal{M}$ in terms of those on $\mathcal{N},(n<d)$,

$$
\begin{aligned}
A_{a, n / 2}^{\mathcal{M}}(p)= & \frac{1}{2 \sqrt{\pi}(D-n)}\left(A_{n / 2}^{\mathcal{N}}(p)+A_{n / 2}^{\mathcal{N}}(p-1)\right) \\
& +\frac{1}{4}\left(A_{(n-1) / 2}^{\mathcal{N}}(p)-A_{(n-1) / 2}^{\mathcal{N}}(p-1)\right) \\
& -\sum_{i=1}^{n-1}\left(A_{(n-1-i) / 2}^{\mathcal{N}}(p) P_{i}\left(z_{a}(p)\right)+A_{(n-1-i) / 2}^{\mathcal{N}}(p-1) P_{i}(x)\right)
\end{aligned}
$$

for absolute conditions.

The $A^{\mathcal{N}}$ are the heat-kernel coefficients corresponding to the base $\zeta-$ function, (15), and the $P_{i}$ are known polynomials arising from the asymptotic expansion of Bessel functions, e.g.

$$
P_{i}(x)=\sum_{b=0}^{i} x_{i, b} \frac{\Gamma((D-n+i) / 2+b)}{\Gamma((D-n+i) / 2) \Gamma(b+i / 2)} .
$$

The $x$ 's correspond to Dirichlet and the $z_{a}(p)$ 's, which depend on $p$ through the $u_{a}(p)$, to Robin conditions.

The combination (22) leads to the total coefficients on $\mathcal{M}$,

$$
A_{a, n / 2}^{\mathcal{M}+}(p)=A_{a, n / 2}^{\mathcal{M}}(p)+A_{a, n / 2}^{\mathcal{M}}(p-1)
$$

and similarly for relative conditions. (31) can be inverted as in (23).

In general $\zeta_{p}^{\mathcal{M}}(s)$ has a pole at $s=0$ which translates into the $\log \tau$ term in the expansion (27), the coefficient being

$$
A_{\mathcal{M}}^{\prime}(p)=-\operatorname{Res} \zeta_{p}^{\mathcal{M}}(0)
$$


These are the only general equations that are needed but the algebra can be checked by confirming Hodge duality on $\mathcal{M}$, in the coefficient form

$$
A_{r, n / 2}^{\mathcal{M}+}(d+1-p)=A_{a, n / 2}^{\mathcal{M}+}(p),
$$

using the easily verified formula,

$$
A_{n / 2}^{\mathcal{N} \pm}(d-p)= \pm A_{n / 2}^{\mathcal{N} \pm}(p)
$$

where, the coefficients $A_{n / 2}^{\mathcal{N} \pm}(p)$ are those resulting from the combinations of the coexact and 'exact' $\zeta$-functions on $\mathcal{N}$ discussed above,

$$
\zeta_{p}^{\mathcal{N} \pm}(s)=\zeta_{p}^{\mathcal{N}}(s) \pm \zeta_{p-1}^{\mathcal{N}}(s)
$$

\section{The ball and sphere $\zeta$-functions.}

We now consider forms on that cone whose base is a unit $d$-sphere, $d=D-1$, i.e. on the $D$-ball. This will be our main application of the general formalism exposed in the previous sections. As we have seen, it is sufficient to look at coexact forms (i.e. transverse, antisymmetric fields).

The form $\zeta$-functions (14) on the $d$-sphere are needed in order to find the corresponding heat-kernel coefficients for substitution into the fundamental equation, (29). The spectral properties have been known for some time $[25,4,29,28,17,36]$ and the coexact $p$-form eigenvalues of the de Rham Laplacian are readily established to be

$$
\mu(p, l)=(l+(d-1) / 2)^{2}-((d-1) / 2-p)^{2}, \quad l=1,2, \ldots .
$$

As anticipated, we again witness the important simplification of the Bessel function order, (5), to the $p$-independent form

$$
\nu(p, l)=l+(d-1) / 2>(d-1) / 2
$$

exactly as in the scalar case. The first consequence is that the absolute $\zeta$-function (24) simplifies to

$$
\zeta_{a, p}^{\text {ball }}(s)=\sum \int_{\gamma} \frac{d k}{2 \pi i} k^{-2 s} \frac{\partial}{\partial k}\left(\tilde{d}(p)\left(\left.\ln \left(r^{u_{a}(p)} \ln J_{\nu}(k r)\right)^{\prime}\right|_{r=1}+d(p-1) \ln J_{\nu}(k)\right) .\right.
$$


For the time being let us work at a fixed $p$. The sphere coexact $p$-form. degeneracy is

$$
d(p, l)=\frac{(2 l+d-1)(l+d-1) !}{p !(d-p-1) !(l-1) !(l+p)(l+d-p-1)} .
$$

We note the symmetry, $d(p, l)=d(d-1-p, l)$ and that $d(d, l)=0$. In addition, there is a zero mode for $p=0$ and one for $p=d$.

Rewrite (38) as

$$
d(p, l)=\frac{(l+d-1) !}{p !(d-p-1) !(l-1) !}\left(\frac{1}{l+p}+\frac{1}{l+d-p-1}\right)
$$

and consider, firstly, the sum

$$
\sum_{l=1}^{\infty} \frac{(l+d-1) !}{(l-1) !} \frac{r^{l+(d-1) / 2}}{l+p}
$$

with $r=\exp (-\tau)<1$. The idea is that this gives the 'square-root' heatkernel, and the sphere $\zeta$-function, (14), follows by Mellin transform on $\tau$ as in our earlier works, $[18,13]$.

The generating function for a given form order can be rewritten using the identity

$$
\sum_{l=1}^{\infty} \frac{(l+d-1) !}{(l-1) !} \frac{r^{l}}{l+p}=(d-p-1) ! \sum_{m=p+1}^{d} \frac{(m-1) !}{(m-p-1) !} \frac{r}{(1-r)^{m}}
$$

which follows easily from recursion.

There is still an overall factor of $r^{(d-1) / 2}$ in (40) and performing the Mellin transform produces a series of Barnes $\zeta$-functions, giving, after the addition of the $p \rightarrow d-p-1$ term, the modified coexact $\zeta$-function, (14), on the sphere as

$$
\begin{aligned}
\zeta_{p}^{S^{d}}(s)= & \sum_{m=p+1}^{d}\left(\begin{array}{c}
m-1 \\
p
\end{array}\right) \zeta_{\mathcal{B}}\left(2 s,(d+1) / 2 \mid \mathbf{1}_{m}\right) \\
& +\sum_{m=d-p}^{d}\left(\begin{array}{c}
m-1 \\
d-p-1
\end{array}\right) \zeta_{\mathcal{B}}\left(2 s,(d+1) / 2 \mid \mathbf{1}_{m}\right)
\end{aligned}
$$

for $0 \leq p<d$. Obviously $\zeta_{d}^{S^{d}}(s)=0$. 
(42) is formally much simpler than expanding the degeneracy to give a series of Hurwitz $\zeta$-functions (a series noted in passing by Copeland and Toms, [17] and frequently used). This will come later.

When $p=0,(42)$, with the zero mode included according to (15), gives the known scalar expression. The first sum reduces to a term that is cancelled by the zero mode and to a single Barnes $\zeta$-function, the result being, [13], [8] eqn. (5.7),

$$
\tilde{\zeta}_{0}^{S^{d}}=\zeta_{\mathcal{B}}\left(2 s ;(d+1) / 2 \mid \mathbf{1}_{d}\right)+\zeta_{\mathcal{B}}\left(2 s ;(d-1) / 2 \mid \mathbf{1}_{d}\right) .
$$

This is elegantly seen from a rearrangement of (42), detailed in Appendix $\mathrm{A}$, that yields $\zeta_{p}^{S^{d}}(s)$ as a finite series of scalar $\zeta$-functions by means of a recursion relating a $p$-form in $d$ dimensions to a $(p-1)$-form in $d-2$. The series is $(0 \leq p<(d-1) / 2)$

$$
\begin{aligned}
\tilde{\zeta}_{p}^{S^{d}}(s)= & \sum_{j=0}^{p}(-1)^{j}\left(\begin{array}{c}
d-1-2 j \\
p-j
\end{array}\right) \tilde{\zeta}_{0}^{S^{d-2 j}}(s) \\
& +\delta_{p, 0}\left(\frac{d-1}{2}\right)^{-2 s}-(-1)^{p}\left(\frac{d-2 p-1}{2}\right)^{-2 s}
\end{aligned}
$$

and this is one of our basic equations. Duality, (19), can be used to extend the range of $p$.

When $d$ is odd we have from (43) the special values for $q \in \mathbb{Z}$,

$$
\tilde{\zeta}_{0}^{S^{d}}(-q)=0, \quad q \geq 0
$$

which corresponds to the fact that for scalars the relevant operator is the (improved) geometrical Laplacian.

From (45) follow the special values of the modified coexact function $(0<$ $p<d)$

$$
\zeta_{p}^{S^{d}}(-q)=\tilde{\zeta}_{p}^{S^{d}}(-q)=(-1)^{p+1}((d-1) / 2-p)^{2 q}
$$

In particular

$$
\zeta_{p}^{S^{d}}(0)=(-1)^{p+1}
$$

and

$$
\zeta_{(d-1) / 2}^{S^{d}}(-q)=0, \quad q>0
$$


Hodge duality, as expressed in (19), can be seen explicitly from (42). Inclusion of the zero modes obviously violates this duality.

It is also worth pointing out that the + combination of coexact and exact $\zeta$-functions in (34) on the sphere, in contrast to (22) on the ball, does not correspond to the $\zeta$-function for any Laplacian.

Finally, we note that $\zeta_{p}^{S^{d}}(s)$ does not have a pole at $s=-1 / 2$, which is important during the construction of the heat-kernel coefficients and the functional determinants on $\mathcal{M}$. It means that the log term is absent in the expansion (27), $c f[8]$. No further discussion of this term is given here. Some relevant comments can be found in the recent works by Bytsenko et al $[10,11]$.

\section{Residues and sphere heat-kernel coefficients.}

To apply (29) to the ball it is necessary to know the coefficients on the sphere. We use (28) on $\mathcal{N}$ with the appropriate $\zeta$-function, in this case either $\zeta^{\mathcal{N}}$ or $\tilde{\zeta}^{\mathcal{N}}$.

From (44), the residue of the modified coexact sphere $\zeta$-function at $s=$ $k / 2, k \in \mathbb{Z}$, is

$$
\operatorname{Res} \zeta_{p}^{S^{d}}(k / 2)=\frac{1}{(k-2) !} \sum_{j=0}^{p}(-1)^{j} \frac{2^{k-d+2 j} D_{d-2 j-k}^{(d-2 j-1)}}{(d-2 j-1)(d-2 j-k) !}\left(\begin{array}{c}
d-2 j-1 \\
p-j
\end{array}\right)
$$

where we have been able to use the results of our earlier work [8] for the residues of 0 -form $\zeta$-functions. The $D_{\nu}^{(n)}$ are easily evaluated generalized Bernoulli polynomials.

The corresponding heat-kernel coefficients are, for $\min ([n / 2], p, d-p)=$ $[n / 2]$ with $d-k=n$,

$$
\begin{aligned}
& \frac{(4 \pi)^{d / 2}}{\left|S^{d}\right|} A_{n / 2}^{S^{d}}(p) \\
& \quad=(k-1) \frac{\Gamma((d+1) / 2)}{\Gamma((k+1) / 2)} \sum_{j=0}^{[n / 2]} \frac{(-1)^{j} 2^{2 j} D_{n-2 j}^{(d-2 j-1)}}{(d-2 j-1)(n-2 j) !}\left(\begin{array}{c}
d-2 j-1 \\
p-j
\end{array}\right) .
\end{aligned}
$$

$A_{n / 2}^{S^{d}}(p)$ vanishes when $n$ is odd and less than $d$ because $D_{\nu}^{(n)}$ is zero for odd $\nu$.

A useful check is provided by the modified coexact coefficients $A_{q+d / 2}^{S^{d}}=$ $(-1)^{q} \zeta_{p}^{S^{d}}(-q) / q !, q \in \mathbb{Z}^{+}$, for odd $d$. According to (46) these half-odd 
coefficients are non-zero, in agreement with the results of [22] and [12] for the de Rham coexact $\zeta$-function. For this case, the only half-odd coefficient is the $q=0$ one, corresponding to $\zeta(0)$, and we know that the modified and de Rham coexact heat-kernels are related by a factor,

$$
K_{p}^{\bmod }(\tau)=e^{-((d-1) / 2-p)^{2} \tau} K_{p}^{\text {de } \operatorname{Rham}}(\tau),
$$

the expansion of which reproduces exactly (46). There are no half-odd coefficients for $n<d$, nor for $p=0$ when the zero mode is included.

Similar statements hold for the 'total' coefficient, $A_{n / 2}^{S^{d}+}$. Because of the construction, (34), of $\zeta_{p}^{S^{d}+}$, one should not, of course, expect a standard geometric form, except when $p=0$.

As a curiosity, constructing the 'total' sphere coefficient $(c f(34))$ by just adding equation (50) for $p$ and $p-1$, we have

$$
\begin{aligned}
& \frac{(4 \pi)^{d / 2}}{\left|S^{d}\right|} A_{n / 2}^{S^{d}+} \\
& =(k-1) \frac{\Gamma((d+1) / 2)}{\Gamma((k+1) / 2)} \sum_{j=0}^{[n / 2]}(-1)^{j} \frac{2^{2 j-n} D_{n-2 j}^{(d-2 j-1)}}{(d-2 j-1)(n-2 j) !}\left(\begin{array}{c}
d-2 j \\
p-j
\end{array}\right),
\end{aligned}
$$

similar to the general form on the ball developed in the next section and also in agreement with the results of Günther and Schimming [27] and Gilkey, e.g. [26].

Equation (51) can be used, in familiar fashion, to give general formulae for the standard, i.e. de Rham, coexact heat-kernel coefficients on the sphere, denoted $B_{n / 2}$, somewhat simpler than those derived in [12] and [22]. Thus

$$
\begin{aligned}
B_{M}^{S^{d}}(p) & =\sum_{N=0}^{M} \frac{w(p)^{M-N}}{(M-N) !} A_{N}^{S^{d}}(p) \\
B_{M+1 / 2}^{S^{d}}(p) & =\sum_{N=0}^{M} \frac{w(p)^{M-N}}{(M-N) !} A_{N+1 / 2}^{S^{d}}(p)
\end{aligned}
$$

where $w(p)=((d-1) / 2-p)^{2}$ and $A_{n / 2}^{S^{d}}(p)$ is given by (50). For odd $d$, the only non-zero half-odd coefficient is $B_{d / 2}^{S^{d}}(p)$, as has already been remarked.

Elizalde et al [22] give selected numerical values for the total $B$ coefficients obtained from a general formula that involves a series of Hurwitz $\zeta$-functions and is slightly less convenient than the above. We have found agreement with their values. 
The connection between the two expressions is simply that the result of [22] follows from ours if the generalized Bernoulli polynomials are expanded in ordinary ones. From a numerical point of view this is unnecessary because there are efficient algorithms that give the generalized functions directly.

\section{General form of coefficients on the ball.}

We now come to the central part of this paper, the explicit construction of the heat-kernel coefficients on the ball.

Firstly we can think of checking against the limited results of [5] for the coefficients for the form-valued Laplacian on manifolds with boundary.

From Theorem 1.2 of [5] applied to the $D$-ball, the total coefficient expressions for absolute boundary conditions are, (the + superscript is dropped in this section)

$$
\begin{aligned}
\frac{(4 \pi)^{d / 2}}{\left|\mathrm{~S}^{d}\right|} A_{0}^{(D)}(p) & =\frac{1}{2 \sqrt{\pi}} \frac{1}{(d+1)}\left(\begin{array}{c}
D \\
p
\end{array}\right) \\
\frac{(4 \pi)^{d / 2}}{\left|\mathrm{~S}^{d}\right|} A_{1 / 2}^{(D)}(p)= & \frac{1}{4}\left[\left(\begin{array}{c}
D \\
p
\end{array}\right)-2\left(\begin{array}{c}
D-1 \\
p-1
\end{array}\right)\right] \\
\frac{(4 \pi)^{d / 2}}{\left|\mathrm{~S}^{d}\right|} A_{1}^{(D)}(p)= & \frac{1}{\sqrt{\pi}} \frac{d}{6}\left[\left(\begin{array}{c}
D \\
p
\end{array}\right)-6\left(\begin{array}{c}
D-1 \\
p-1
\end{array}\right)+6\left(\begin{array}{c}
D-2 \\
p-2
\end{array}\right)\right] \\
\frac{(4 \pi)^{d / 2}}{\left|\mathrm{~S}^{d}\right|} A_{3 / 2}^{(D)}(p)= & \frac{d}{384}\left[(13 d+2)\left(\begin{array}{c}
D \\
p
\end{array}\right)-4(29 d-26)\left(\begin{array}{c}
D-1 \\
p-1
\end{array}\right)\right. \\
& \left.\quad+288(d-1)\left(\begin{array}{c}
D-2 \\
p-2
\end{array}\right)-192(d-1)\left(\begin{array}{c}
D-3 \\
p-3
\end{array}\right)\right] .
\end{aligned}
$$

Assuming, according to our general philosophy, that $d$ is sufficiently large, it has been checked that the first three coefficients follow from our formulation using (29), (50) and various binomial identities including the recursion

$$
\left(\begin{array}{l}
a \\
b
\end{array}\right)=\left(\begin{array}{c}
a-1 \\
b
\end{array}\right)+\left(\begin{array}{l}
a-1 \\
b-1
\end{array}\right)
$$

The fourth coefficient will be confirmed later by a more powerful technique, and the series continued.

Although these results were derived on the basis of a restriction on $p$, no such restriction exists for the expressions (54) in [5]. The conclusion is that, once the coefficients are displayed as explicit functions of $d$ and $p$, they can 
be continued outside the restrictions, the reason being that their particular structure is guaranteed by the geometric formulation.

These particular checks show that our general formulae are correct but it is not feasible to pursue the further evaluation of the coefficients in this way by algebraic rearrangement of the produced expressions, since it involves awkwardly repeated application of the binomial recursion and of other identities. A systematic approach to the evaluation of any coefficient is better provided by fitting unknowns in a general form, and this process will now be set in train.

From basic theory (e.g. [26]) the geometric expression on a flat, bounded $D$-manifold, $\mathcal{M}$, is, up to terms involving derivatives of the extrinsic curvature $\kappa$,

$$
c(n)(4 \pi)^{d / 2} A_{n / 2}^{D}(p)=\int_{\partial \mathcal{M}} b_{\mathbf{n}}(D, p) \sum_{\mathbf{n}}\left(\operatorname{tr}\left(\kappa^{n_{1}}\right) \operatorname{tr}\left(\kappa^{n_{2}}\right) \ldots\right)
$$

with

$$
\begin{aligned}
c(n) & =2 \sqrt{\pi}, \quad n \text { even } \\
& =1, \quad n \text { odd } \\
& =2(d+1) \sqrt{\pi}, \quad n=0
\end{aligned}
$$

and where $\mathbf{n}=\left(n_{1}, n_{2}, \ldots\right)$ is a partition of $n-1$. For convenience the $n=0$ term has been included although it is really a volume contribution. We seek the $b_{\mathbf{n}}(D, p)$.

For the $D$-ball, (56) reduces to

$$
c(n)(4 \pi)^{d / 2} A_{n / 2}^{D}(p)=\left|\mathrm{S}^{d}\right| \sum_{\mathbf{n}} d^{|\mathbf{n}|} b_{\mathbf{n}}(D, p)=\left|\mathrm{S}^{d}\right| \sum_{k=1}^{n-1} d^{k} b_{k}^{(n)}(D, p)
$$

where $|\mathbf{n}|$ is the number of components in the partition and $b_{k}^{(n)}$, is the sum of those $b_{\mathbf{n}}$ for which $|\mathbf{n}|=k$,

$$
b_{k}^{(n)}(D, p)=\left.\sum_{\mathbf{n}} b_{\mathbf{n}}(D, p)\right|_{|\mathbf{n}|=k} .
$$

These are the only combinations that can be determined from working on the ball.

The numerical multipliers $b_{\mathbf{n}}(D, p)$ satisfy the binomial recursion

$$
b_{\mathbf{n}}(D, p)=b_{\mathbf{n}}(D-1, p)+b_{\mathbf{n}}(D-1, p-1),
$$


proved by crossing $\mathcal{M}$ with a unit circle, [5]. This relation is what has become of the more familiar statement of dimension-independence for scalars.

Thus $b_{\mathbf{n}}(D, p)$ can be expanded as a linear combination of binomial coefficients $\left(\begin{array}{c}D+a \\ p+b\end{array}\right)$ for varying $a$ and $b$. Since $b_{\mathbf{n}}(D, p)$ vanishes for $p$ outside the range 0 to $D, a$ must equal $b$ and be nonpositive. Consequently the expansion reads

$$
b_{\mathbf{n}}(D, p)=\sum_{m=0}^{n} M_{\mathbf{n}, m}\left(\begin{array}{c}
D-m \\
p-m
\end{array}\right)
$$

which is the boundary version of the Günther and Schimming form [27]. The limits have been fixed by noting that they must be independent of both $D$ and $p$ and, therefore, can be set by considering the particular value $D=n$, for which there are $n+1$ independent constants implying $n+1$ terms in the sum. The trivial first coefficient, $b_{0}=\left(\begin{array}{l}D \\ p\end{array}\right)$, can also be used to pin down the limits to those shown. The conclusion is that, on the ball, the general form can be written,

$$
\frac{c(n)(4 \pi)^{d / 2}}{\left|S^{d}\right|} A_{n / 2}^{(D)}(p)=\sum_{m=0}^{n} P_{m}^{(n)}(d)\left(\begin{array}{c}
D-m \\
p-m
\end{array}\right)
$$

where $P_{m}^{(n)}(d)$ is a polynomial of degree $n-1$ in $d$. For $n>1$

$$
P_{m}^{(n)}(d)=\sum_{\mathbf{n}} M_{\mathbf{n}, m} d^{|\mathbf{n}|}=\sum_{k=1}^{n-1} M_{k, m}^{(n)} d^{k}
$$

where the $M_{k, m}^{(n)}$ are constants.

Our method is simply that, for fixed $n$, knowing the left-hand side for $0 \leq p \leq n,(60)$ can be inverted for the $P_{m}^{(n)}(d)$, keeping $d$ unspecified. The method employed in [5] uses the particular values $b_{\mathbf{n}}(n, p)$ to invert (59). Our technique is more flexible in practice, although the analysis in [5] does bring out clearly the amusing fact that the general coefficient can be generated from just the values $A_{n / 2}^{(n)}(p), 0 \leq p \leq n$.

In detail, we firstly note that, for $p=0$ to $n$, the matrix of binomial coefficients on the right-hand side of (60) is triangular so that the inversion follows recursively by forward substitution,

$$
P_{m}^{(n)}(d)=\frac{c(n)(4 \pi)^{d / 2}}{\left|\mathrm{~S}^{d}\right|} A_{n / 2}^{(D)}(m)-\sum_{\mu=0}^{m-1}\left(\begin{array}{c}
D-\mu \\
m-\mu
\end{array}\right) P_{\mu}^{(n)}(d), \quad m=0, \ldots, n .
$$


The driving coefficients $A_{n / 2}^{(D)}(m)(0 \leq m \leq n)$ will be determined from (29) as polynomials in $d$ because, for given numerical values of $p$ and $n$, the sphere coefficients, (50), are obviously such polynomials.

It should be stated that the $\zeta$-function on the ball is a standard one, and that the heat-kernel coefficients are geometric (leading to the general form (60)). In this case it is quite permissible to evaluate the polynomials in whatever parameter region is convenient and then continue, despite the fact that they involve coefficients on the sphere for which this might not be possible.

Evaluation is a routine machine matter, the first outcome of which is $A_{3 / 2}^{(D)}(p)$ in (54). Some results are given below in the form of matrices of the constants $M_{k, m}^{(n)}$ in (61),

$$
\mathbf{M}_{a}^{(3)}=\left(\begin{array}{cccc}
\frac{1}{192} & \frac{13}{48} & -\frac{3}{4} & \frac{1}{2} \\
\frac{13}{384} & -\frac{29}{96} & \frac{3}{4} & -\frac{1}{2}
\end{array}\right)
$$

(64)

(65)

$$
\mathbf{M}_{a}^{(4)}=\left(\begin{array}{ccccc}
\frac{4}{135} & -\frac{164}{315} & \frac{16}{5} & -\frac{16}{3} & \frac{8}{3} \\
\frac{1}{45} & \frac{92}{105} & -\frac{74}{15} & 8 & -4 \\
\frac{1}{27} & -\frac{136}{315} & \frac{26}{15} & -\frac{8}{3} & \frac{4}{3}
\end{array}\right)
$$

$$
\mathbf{M}_{a}^{(5)}=\left(\begin{array}{cccccc}
\frac{77}{15360} & \frac{77}{960} & -\frac{191}{192} & \frac{19}{6} & -\frac{15}{4} & \frac{3}{2} \\
\frac{235}{36864} & -\frac{263}{1440} & \frac{1475}{768} & -\frac{47}{8} & \frac{55}{8} & -\frac{11}{4} \\
\frac{139}{61440} & \frac{1987}{15360} & -\frac{1769}{1536} & \frac{157}{48} & -\frac{15}{4} & \frac{3}{2} \\
\frac{2041}{737280} & -\frac{3787}{92160} & \frac{347}{1536} & -\frac{9}{16} & \frac{5}{8} & -\frac{1}{4}
\end{array}\right)
$$

A subscript has been added to indicate that these values are for absolute boundary conditions. Although any relative coefficient can be found from 
the absolute ones using duality, in order to obtain the general form the entire analysis should be repeated. A tactically better way combines these procedures and consists of using the recursive nature of the relative coefficients to write down a general form, precisely as in (60), together with the corresponding solution, (62), but now where the values of the coefficients for $p=0$ to $n$ are determined by duality in terms of already evaluated absolute quantities. In any case, one rapidly finds that the first coefficient is the same, that the second is reversed in sign and that the remaining ones (up to $n=5$ ) are contained in the matrices,

$$
\begin{aligned}
\mathbf{M}_{r}^{(3)} & =\left(\begin{array}{cccc}
\frac{5}{192} & -\frac{13}{48} & \frac{3}{4} & -\frac{1}{2} \\
-\frac{7}{384} & \frac{29}{96} & -\frac{3}{4} & \frac{1}{2}
\end{array}\right) \\
\mathbf{M}_{r}^{(4)} & =\left(\begin{array}{ccccc}
\frac{8}{189} & -\frac{172}{315} & \frac{16}{5} & -\frac{16}{3} & \frac{8}{3} \\
-\frac{11}{315} & \frac{104}{105} & -\frac{74}{15} & 8 & -4 \\
\frac{1}{189} & -\frac{116}{315} & \frac{26}{15} & -\frac{8}{3} & \frac{4}{3}
\end{array}\right)
\end{aligned}
$$

(66)

$$
\mathbf{M}_{r}^{(5)}=\left(\begin{array}{cccccc}
\frac{109}{15360} & -\frac{29}{320} & \frac{193}{192} & -\frac{19}{6} & \frac{15}{4} & -\frac{3}{2} \\
-\frac{1049}{184320} & \frac{1247}{5760} & -\frac{1501}{768} & \frac{47}{8} & -\frac{55}{8} & \frac{11}{4} \\
\frac{47}{61440} & -\frac{709}{5120} & \frac{1783}{1536} & -\frac{157}{48} & \frac{15}{4} & -\frac{3}{2} \\
\frac{13}{147456} & \frac{2467}{92160} & -\frac{325}{1536} & \frac{9}{16} & -\frac{5}{8} & \frac{1}{4}
\end{array}\right)
$$

It is a matter of a few minutes by machine algebra to calculate larger matrices.

A direct relation between the absolute and relative quantities can be obtained algebraically. The $M$-matrix elements are connected by

$$
M_{r ; k, m}^{(n)}=(-1)^{m} \sum_{i=m}^{n}\left(\begin{array}{c}
i \\
m
\end{array}\right) M_{a ; k, i}^{(n)}
$$


by virtue of the identity

$$
\left(\begin{array}{c}
D-i \\
p
\end{array}\right)=\sum_{m=0}^{i}(-1)^{m}\left(\begin{array}{c}
i \\
m
\end{array}\right)\left(\begin{array}{c}
D-m \\
p-m
\end{array}\right)
$$

easily proved by induction on $i$.

Knowledge of these constants enables restrictions to be placed on the multipliers, $b_{\mathbf{n}}(D, p)$, in the general geometric form of the coefficients, (56).

Denoting the set of $b_{k}^{(n)}(D, p),(1 \leq k \leq n-1)$, by the vector $\mathbf{b}^{(n)}$, the vector of binomial coefficients $\left(\begin{array}{c}D-m \\ p-m\end{array}\right),(0 \leq m \leq n)$, by $\mathbf{c}^{(n)}$ and the set of powers $d^{k}(1 \leq k \leq n-1)$ by $\mathbf{d}^{(\mathbf{n})}$, equation (60) takes the matrix form

$$
\frac{c(n)(4 \pi)^{d / 2}}{\left|\mathrm{~S}^{d}\right|} A_{n / 2}^{(D)}(p)=\widetilde{\mathbf{d}^{(n)}} \mathbf{M}^{(n)} \mathbf{c}^{(n)}
$$

and the restrictions are

$$
\mathbf{b}^{(n)}=\mathbf{M}^{(n)} \mathbf{c}^{(n)}
$$

For example, for $n=5$ there are 4 partitions so

$$
b_{1}^{(5)}=b_{4}, \quad b_{2}^{(5)}=b_{1,3}+b_{2^{2}}, \quad b_{3}^{(5)}=b_{1^{2}, 2}, \quad b_{4}^{(5)}=b_{1^{4}}
$$

and only these particular combinations are known using (69) and (65) or (66). It is clear that one can always unambiguously determine $b_{n-1}^{(n)}$ and $b_{1^{n-1}}^{(n)}$.

We finally note that the $n=4$ values at $D=4$ agree with those in Table 2 of Moss and Poletti [32].

\section{Generating functions.}

A generating function approach to the form order, $p$, could be introduced at this coefficient stage by defining

$$
\begin{aligned}
\bar{A}_{n / 2}^{(D)}(\sigma) & =\sum_{p=0}^{D}(-\sigma)^{p} A_{n / 2}^{(D)}(p) \\
& =\frac{\left|\mathrm{S}^{d}\right|}{c(n)(4 \pi)^{d / 2}} \sum_{m=0}^{n}(-\sigma)^{m}(1-\sigma)^{D-m} P_{m}^{(n)}(d) .
\end{aligned}
$$


In particular the value $\sigma=-1$ is required ( $c f[5] \mathrm{Thm} .1 .3$ ),

$$
\bar{A}_{n / 2}^{(D)}(-1)=\frac{4 \sqrt{\pi}}{c(n) \Gamma((d-1) / 2)} \sum_{m=0}^{n} 2^{-m} P_{m}^{(n)}(d)
$$

which is a convenient way of organising and computing this quantity, at this point.

There is topological interest in the case $\sigma=1$ and we see from (70) that

$$
\begin{aligned}
& \bar{A}_{a, n / 2}^{(D)}(1)=0, \quad n<D ; \\
& \bar{A}_{a, D / 2}^{(D)}(1)=\frac{(-1)^{D} 2^{1-d} \sqrt{\pi}}{c(D) \Gamma((d-1) / 2)} P_{D}^{(D)}(d)=\chi(\mathcal{M})=1
\end{aligned}
$$

which provides a check of the Gauss-Bonnet theorem. If relative coefficients are used we find $\chi(\mathcal{M}, \partial \mathcal{M})=-1$, which is correct.

\section{Value at zero.}

Special interest, both mathematical and physical, attaches itself to the particular value $\zeta_{p}^{\mathcal{M}+}(0)$ which merits individual treatment, e.g. $[32,24,3]$. Further, as already said, these values play an important role in the algebraic computations of Blažić et al [5].

Generally, for absolute conditions, the coexact expression is

$$
\begin{gathered}
\zeta_{p}^{\mathcal{M}}(0)=-\frac{1}{2}\left(\tilde{\zeta}_{p}^{\mathcal{N}}(-1 / 2)+\zeta_{p-1}^{\mathcal{N}}(-1 / 2)\right)+\frac{1}{4}\left(\tilde{\zeta}_{p}^{\mathcal{N}}(0)-\zeta_{p-1}^{\mathcal{N}}(0)\right) \\
-\sum_{k=1}^{d} \frac{1}{k}\left(\zeta_{R}(-k)\left(\operatorname{Res} \zeta_{p}^{\mathcal{N}}(k / 2)+\operatorname{Res} \zeta_{p-1}^{\mathcal{N}}(k / 2)\right)\right. \\
\left.-\left(-u_{a}(p)\right)^{k} \operatorname{Res} \zeta_{p}^{\mathcal{N}}(k / 2)\right)
\end{gathered}
$$

For $\mathcal{N}=\mathrm{S}^{d}$ the residues are contained in (49) and the values of the $\zeta$ function found from (42) or (44) (there are no zero modes on the sphere in the sense that $\nu>0)$.

An alternative evaluation consists of using the standard formula

$$
\zeta_{p}^{\mathcal{M}+}(0)=A_{D / 2}^{(D)}(p)-\beta_{p}^{\mathcal{M}}
$$

together with an explicitly computed general coefficient (60). The absolute Betti numbers of the ball are $\beta_{0}=1$, the rest vanishing. 
The direct evaluation via (73) is the most efficient, numerically. Machine computation shows agreement between these two approaches and also with the values presented in [21], except that the $p=0$ numbers for $d=6$ and 7 differ.

It is a remarkable fact that in using (29), leading to (60) and thence to (74), one does not have to worry about the 'zero modes' in say (15), whilst such contributions are vital when evaluating directly from (73).

Finally, using the inverse $(23)$ and noting that $\zeta_{D}^{\mathcal{M}}(0)$ vanishes, we reconfirm the topological formula

$$
\chi=\sum_{p=0}^{D}(-1)^{p} \beta_{p}^{\mathcal{M}}=\sum_{p=0}^{D}(-1)^{p} A_{D / 2}^{D}(p) .
$$

\section{Form functional determinants.}

In this section we consider functional determinants for $p$-forms. As we have seen in the calculation of the heat-kernel coefficients, it will be enough to consider the determinant associated with the coexact $\zeta$-function (24), this being simply a combination of Robin and Dirichlet contributions. It is immediately appreciated that the results derived in [8], eq. (9.8) for Dirichlet boundary conditions and eq. (11.2) for Robin conditions, remain valid once the base $\zeta$-function there is replaced with the $p$-form base $\zeta$-function, eq. (14), with, in addition,

$$
\zeta_{p}^{\mathcal{N}+1}(s)=\sum_{n=1}^{\infty} \sum \frac{d(p)}{(\nu(p)+n)^{s}}, \quad \text { and } \quad \zeta_{p}^{\mathcal{N}}(s, u)=\sum \frac{d(p)}{(\nu(p)+u)^{s}},
$$

and the related quantities, $\tilde{\zeta}_{p}^{\mathcal{N}+1}(s), \tilde{\zeta}_{p}^{\mathcal{N}}(s, u)$, if the zero modes are included as in (15).

The coexact determinant for $p \geq 1$ combines Robin with Dirichlet and is determined, for absolute conditions, by the derivatives,

$$
\begin{aligned}
\zeta_{p}^{\prime \mathcal{M}}(0)= & \tilde{\zeta}_{p}^{\prime \mathcal{N}+1}(0)+\zeta_{p-1}^{\mathcal{N}+1}(0)+\tilde{\zeta}_{p}^{\prime \mathcal{N}}\left(0, u_{a}(p)\right) \\
& +\ln 2\left(\tilde{\zeta}_{p}^{\mathcal{N}}(-1 / 2)+\zeta_{p-1}^{\mathcal{N}}(-1 / 2)\right. \\
& +2 \sum_{\substack{i=1 \\
i \text { odd }}}^{d} \operatorname{Res} \zeta_{p}^{\mathcal{N}}(i / 2) M_{i}\left(1, u_{a}(p)\right)
\end{aligned}
$$




$$
\begin{gathered}
\left.+2 \sum_{i=1}^{d} \operatorname{Res} \zeta_{p-1}^{\mathcal{N}}(i / 2) D_{i}(1)\right) \\
+2 \sum_{\substack{i=1 \\
i \text { odd }}}^{d} \operatorname{Res} \zeta_{p}^{\mathcal{N}}(i / 2)\left(M_{i}\left(1, u_{a}(p)\right) \sum_{k=1}^{i-1} 1 / k\right. \\
\left.\quad+\int_{0}^{1} d t \frac{M_{i}\left(t, u_{a}(p)\right)-t M_{i}\left(1, u_{a}(p)\right)}{t\left(1-t^{2}\right)}\right) \\
+2 \sum_{\substack{i=1 \\
i \text { even }}}^{d} \operatorname{Res} \zeta_{p}^{\mathcal{N}}(i / 2)\left(M_{i}\left(1, u_{a}(p)\right) \sum_{k=1}^{i-1} 1 / k\right. \\
\left.+\int_{0}^{1} d t \frac{M_{i}\left(t, u_{a}(p)\right)-t^{2} M_{i}\left(1, u_{a}(p)\right)}{t\left(1-t^{2}\right)}\right) \\
+2 \sum_{\substack{i=1 \\
d}}^{\operatorname{Res}} \zeta_{p-1}^{\mathcal{N}}(i / 2)\left(D_{i}(1) \sum_{k=1}^{i-1} 1 / k\right. \\
\left.+\int_{0}^{1} d t \frac{D_{i}(t)-t D_{i}(1)}{t\left(1-t^{2}\right)}\right) .
\end{gathered}
$$

The $M_{i}$ and $D_{i}$ are known polynomials associated with the asymptotic expansion of Bessel functions and, to avoid repetition, we refer to [8], and to references therein, for more details. Some information can be found incidentally in Appendix D.

For $p=0$ a small extra consideration is necessary. As we have mentioned, absolute 0 -forms are Neumann scalars and the Robin parameter is then $u_{a}(0)=-(d-1) / 2$. Looking at $\zeta_{p}^{\mathcal{N}}(s, u)$ in eq. (75) it is seen that, for $u=$ $-\nu(p)$, one encounters a branch cut occasioned by (incorrectly) including the true zero mode for Neumann conditions. The technical reason is that the asymptotic expansion for these specific Robin parameters is slightly different from the others.

The easiest way of taking this into account is to subtract the contribution in (75) for $u \neq-\nu(p)$, then take the limit as $u \rightarrow-\nu(p)$ and finally to add the correct contribution for $u=-\nu(p)$.

For Neumann conditions the end result is

$$
\begin{aligned}
\zeta_{0}^{\prime \mathcal{M}}(0)= & \tilde{\zeta}_{0}^{\prime \mathcal{N}+1}(0) \\
& +\ln 2\left(\tilde{\zeta}_{0}^{\mathcal{N}}(-1 / 2)+2 \sum_{\substack{i=1 \\
i \text { odd }}}^{d} \operatorname{Res} \zeta_{0}^{\mathcal{N}}(i / 2) M_{i}\left(1, u_{a}(0)\right)\right)
\end{aligned}
$$




$$
\begin{aligned}
& +2 \sum_{\substack{i=1 \\
i \text { odd }}}^{d} \operatorname{Res} \zeta_{0}^{\mathcal{N}}(i / 2)\left(M_{i}\left(1, u_{a}(0)\right) \sum_{k=1}^{i-1} 1 / k\right. \\
& \left.\quad+\int_{0}^{1} d t \frac{M_{i}\left(t, u_{a}(0)\right)-t M_{i}\left(1, u_{a}(0)\right)}{t\left(1-t^{2}\right)}\right) \\
& +2 \sum_{\substack{i=1 \\
i \text { even }}}^{d} \operatorname{Res} \zeta_{0}^{\mathcal{N}}(i / 2)\left(M_{i}\left(1, u_{a}(0)\right) \sum_{k=1}^{i-1} 1 / k\right. \\
& \left.+\int_{0}^{1} d t \frac{M_{i}\left(t, u_{a}(0)\right)-t^{2} M_{i}\left(1, u_{a}(0)\right)}{t\left(1-t^{2}\right)}\right) \\
& +\lim _{u \rightarrow-(d-1) / 2}\left(\tilde{\zeta}_{0}^{\prime \mathcal{N}}(0, u)+\ln ((d-1) / 2+u)\right)+\ln (d+1) .
\end{aligned}
$$

The relative, Dirichlet expression is given in [8].

Equations (76) and (77) are expressions on the generalized cone and we apply them now to the ball, for which all quantities have already been treated, apart from $\zeta_{p}^{\mathcal{N}+1}(s)\left(\tilde{\zeta}_{p}^{\mathcal{N}+1}(s)\right)$ and $\zeta_{p}^{\mathcal{N}}(s, u)\left(\tilde{\zeta}_{p}^{\mathcal{N}}(s, u)\right)$. For these remaining $\zeta$-functions one immediately finds, along the lines of [8],

$$
\begin{aligned}
\tilde{\zeta}_{p}^{\mathcal{N}+1}(s)= & \left.\sum_{m=p+1}^{d}\left(\begin{array}{c}
m-1 \\
p
\end{array}\right) \zeta_{\mathcal{B}}\left(s,(d+3) / 2 \mid \mathbf{1}_{m+1}\right)+\zeta_{H}(s ;(d+3) / 2)\right) \delta_{p d} \\
& \left.+\sum_{m=d-p}^{d}\left(\begin{array}{c}
m-1 \\
d-p-1
\end{array}\right) \zeta_{\mathcal{B}}\left(s,(d+3) / 2 \mid \mathbf{1}_{m+1}\right)+\zeta_{H}(s ;(d+1) / 2)\right) \delta_{p 0}
\end{aligned}
$$

and

$$
\begin{aligned}
\tilde{\zeta}_{p}^{\mathcal{N}}\left(s, u_{a}\right)= & \sum_{m=p+1}^{d}\left(\begin{array}{c}
m-1 \\
p
\end{array}\right) \zeta_{\mathcal{B}}\left(s, p+1 \mid \mathbf{1}_{m}\right) \\
& +\sum_{m=d-p}^{d}\left(\begin{array}{c}
m-1 \\
d-p-1
\end{array}\right) \zeta_{\mathcal{B}}\left(s, p+1 \mid \mathbf{1}_{m}\right) \\
& +\delta_{p 0}((d-1) / 2+u)^{-s}+\delta_{p d}(d+1)^{-s}
\end{aligned}
$$

The contribution of the zero modes is visible and $\zeta_{p}^{\mathcal{N}+1}(s), \zeta_{p}^{\mathcal{N}}\left(s, u_{a}\right)$ need not be stated. It is clearly seen that the limit $u \rightarrow-(d-1) / 2$ in $(77)$ is well defined because the logarithm is cancelled by the last term in (79). 
Using (78) and (79) in (76) and (77), the determinants emerge as derivatives of the Barnes $\zeta$-function at $s=0$. These may be expanded using Stirling numbers,

$$
\begin{aligned}
\zeta_{\mathcal{B}}\left(s, a \mid \mathbf{1}_{m}\right) & =\sum_{n=0}^{\infty}\left(\begin{array}{r}
m+n-1 \\
m-1
\end{array}\right)(a+n)^{-s} \\
& =\sum_{k=1}^{m} \frac{(-1)^{k+m}}{(k-1) !(m-k) !} B_{m-k}^{(m)}(a) \zeta_{H}(s+1-k, a)
\end{aligned}
$$

to give derivatives of Hurwitz or Riemann $\zeta$-functions, [2] p.433. The derivatives presented in Appendix B were obtained in this way. It is easy to find the expression in any dimension $d$ and for any value of $p$.

The presentation of our results is a little more explicit than the corresponding ones of [21] in that only derivatives of Riemann $\zeta$-functions are involved, there being no integrals over $\Gamma$ or $\Psi$ functions. In fact, the integrals in [21] can be done, bringing exact agreement between the two sets of expressions. This agreement is elaborated, in its essentials, in Appendix C.

It should be noted that, as a consequence of an earlier error in [7] for Neumann 0 -forms, a $\log 2$ term should be added to the results in [21] for $p=0$ and $d \geq 2$.

\section{Spectral properties of spinors.}

For the sake of completeness, the method of [8] is now employed to put the results of the earlier work on spinors, [19], on a secure and systematic footing. For any undefined quantities and for further explanations, refer to this paper.

\section{Spectral boundary conditions.}

In [19] it was shown that spectral boundary conditions lead to

$$
J_{n+D / 2-1}(k)=0, \quad n \in \mathbb{Z},
$$

with the degeneracy

$$
d(n)=2 d_{S}\left(\begin{array}{c}
n+D-2 \\
n
\end{array}\right) .
$$


Having in mind the treatment of the scalar field in [8] we define the spinor base $\zeta$-function appropriate for the ball,

$$
\zeta_{\mathcal{N}}(s)=\sum_{n=0}^{\infty} d(n)(n+D / 2-1)^{-2 s} .
$$

This choice guarantees that all formulas in terms of $\zeta_{\mathcal{N}}$ in section 3 of [8] remain valid. Also the polynomials $D_{i}(t)$ defined there are unchanged. In particular, eq. (4.8) is still true, the only difference is in the base $\zeta$-function used for the calculation of $A_{n / 2}^{\mathcal{N}}$, the base heat-kernel coefficients.

Let us continue with the treatment of $\zeta_{\mathcal{N}}(s)$ for the ball. It has the especially simple form

$$
\zeta_{\mathcal{N}}(s)=2 d_{S} \zeta_{\mathcal{B}}\left(2 s,(d-1) / 2 \mid \mathbf{1}_{d}\right) .
$$

To obtain the heat-kernel coefficients using eq. (4.8) of [8], we need only the residues in (84). Using the known values of the Barnes $\zeta$-function in eq. (4.8) of [8] one regains all the results of [19] for the heat-kernel coefficients including the conjecture for their general form.

The determinants come out similarly. Due to the identical structure of the present problem, eq. (9.8) of [8] remains valid once the base zeta function there is replaced by (83). The only 'unknown' quantity is

$$
\zeta_{\mathcal{N}+1}(s)=2 d_{S} \zeta_{\mathcal{B}}\left(s,(d+1) / 2 \mid \mathbf{1}_{d+1}\right)
$$

Using (80) this can be expanded in Hurwitz-Riemann $\zeta$-functions, and complete agreement with the results exhibited in [19] is again quickly and economically found.

In Appendix D we prove the curious identity for the spectral heat-kernel coefficients,

$$
A_{(D-2) / 2}^{(S)}(D)=0, \quad D \text { even, }
$$

observed numerically in [19], the significance of which has not yet been discovered.

\section{Local boundary conditions.}

For local boundary conditions a few more technical details are necessary. The eigenvalue condition is, [19],

$$
J_{n+D / 2}^{2}(k)-J_{n+D / 2-1}^{2}(k)=0, \quad n \in \mathbb{Z}
$$


with the mode degeneracy

$$
d(n)=d_{S}\left(\begin{array}{c}
n+D-2 \\
n
\end{array}\right),
$$

which is half that of the spectral case. Defining the base $\zeta$-function as in (83) we have

$$
\zeta_{\mathcal{N}}(s)=d_{S} \zeta_{\mathcal{B}}\left(2 s, D / 2-1 \mid \mathbf{1}_{d}\right) .
$$

The polynomials describing the asymptotics are a bit different, however, and read

$$
D_{i}(t)=\sum_{a=0}^{2 i} x_{i, a} t^{i+a},
$$

which leads to a slightly different form of the $A_{i}(s)$. These are found to be (one only has to refer to [30] eq. (2.18), and replace the $\zeta$-functions by the base $\zeta$-function),

$$
\begin{aligned}
A_{-1}(s) & =\frac{1}{2 \sqrt{\pi}} \frac{\Gamma(s-1 / 2)}{\Gamma(s+1)} \zeta_{\mathcal{N}}(s-1 / 2) \\
A_{0}(s) & =-\frac{1}{2 \sqrt{\pi}} \frac{\Gamma(s+1 / 2)}{\Gamma(s+1)} \zeta_{\mathcal{N}}(s) \\
A_{i}(s) & =-\frac{1}{\Gamma(s)} \zeta_{\mathcal{N}}(s+i / 2) \sum_{a=0}^{2 i} x_{i, a} \frac{\Gamma(s+(i+a) / 2)}{\Gamma((i+a) / 2)} .
\end{aligned}
$$

Due to the different $A_{0}(s)$ and $A_{i}(s)$ the heat-kernel coefficients this time read

$$
\begin{aligned}
A_{n / 2}^{\mathcal{M}}= & \frac{\Gamma((D-n-1) / 2)}{\sqrt{\pi}(D-n)} \operatorname{Res} \zeta_{\mathcal{N}}((D-n-1) / 2) \\
& -\frac{\Gamma((D-n+1) / 2)}{\sqrt{\pi}(D-n)} \operatorname{Res} \zeta_{\mathcal{N}}((D-n) / 2) \\
& -\sum_{i=1}^{n-1} \operatorname{Res} \zeta_{\mathcal{N}}((D-n+i) / 2) \sum_{a=0}^{2 i} x_{i, a} \frac{\Gamma((D-n+i+a) / 2)}{\Gamma((i+a) / 2)}
\end{aligned}
$$

It would be possible to rewrite the right-hand side in terms of the spinor heat-kernel coefficients on the base, leading to an equation like (29).

For the ball, using (49) for the residues of $\zeta_{\mathcal{N}}(s)$, (89), all results of [19] are immediately reproduced in an organised and rapid fashion. Let us therefore proceed to the determinant. 
As a result of (90) one has

$$
\begin{aligned}
A_{-1}^{\prime}(0) & =2(\ln 2-1) \zeta_{\mathcal{N}}(-1 / 2)-\zeta_{\mathcal{N}}^{\prime}(-1 / 2), \\
A_{0}^{\prime}(0) & =\ln 2 \zeta_{\mathcal{N}}(0)-\frac{1}{2} \zeta_{\mathcal{N}}^{\prime}(0), \\
A_{i}^{\prime}(0) & =-\sum_{a=0}^{2 i} x_{i, a}\left(P P \zeta_{\mathcal{N}}(i / 2)+\gamma \operatorname{Res} \zeta_{\mathcal{N}}(i / 2)+\right. \\
& \left.\operatorname{Res} \zeta_{\mathcal{N}}(i / 2) \psi((a+i) / 2)\right),
\end{aligned}
$$

with characteristic differences to the spectral case. Further contributions come from

$$
\mathcal{Z}^{\prime}(0)=\sum_{n=0}^{\infty} d(n) \mathcal{Z}^{\nu^{\prime}}(0), \quad \nu=n+D / 2-1
$$

and

$$
\mathcal{Z}^{\nu^{\prime}}(0)=2 \ln \Gamma(\nu+1)+2 \nu-2 \nu \ln \nu-\ln (2 \pi \nu)+\sum_{n=1}^{D-1} \frac{D_{n}(1)}{\nu^{n}} .
$$

This follows from the results preceding eq. (3.1) in [30].

Formally (94) is twice the scalar case. It also shows that $D_{n}(1)=$ $2 \zeta_{R}(-n) / n$ and so the contribution $\mathcal{Z}^{\prime}(0)$ is known from the scalar field calculation. Adding up, everything fits nicely and the final answer reads

$$
\begin{aligned}
\zeta_{1 / 2}^{l o}{ }^{\prime}(0)= & \ln 2\left(2 \zeta_{\mathcal{N}}(-1 / 2)+\zeta_{\mathcal{N}}(0)\right)+2 \zeta_{\mathcal{N}+1}^{\prime}(0) \\
& +4 \ln 2 \sum_{i=1}^{D-1} \frac{\zeta_{R}(-i)}{i} \operatorname{Res} \zeta_{\mathcal{N}}(i / 2) \\
& +2 \sum_{i=1}^{D-1} \operatorname{Res} \zeta_{\mathcal{N}}(i / 2)\left[\frac{2 \zeta_{R}(-i)}{i} \sum_{k=1}^{i-1} \frac{1}{k}+\int_{0}^{1} d t \frac{D_{i}(t)-t D_{i}(1)}{t\left(1-t^{2}\right)}\right]
\end{aligned}
$$

Specialising to the ball, all results of [19] are thereby established again in a systematic and general way.

\section{Conclusion.}

Our essential results are, firstly, the expression (29) for the coexact heatkernel coefficients on the generalized cone in terms of those on its base. Secondly, (60) gives any ball heat-kernel coefficient, with explicitly computable 
polynomials, (61), and leads to the restriction (69) on the general coefficients. Thirdly, we draw attention to the formula (76) for the functional determinant and finally to the expression (44) for the modified $p$-form coexact $\zeta$-function on the sphere as a finite sum of (conformal) 0 -form functions.

To save excessive analysis, we have concentrated on the simplest generalized cone, i.e. the ball. As a continuation, more complicated bases, $\mathcal{N}$, can be considered. In the earlier paper, [8], we looked at the case when the base was a sphere of non-unit radius, say $a$, calling this a monopole. Then, in place of (36) for the order of the Bessel functions, we have

$$
\nu^{2}(l, p)=\frac{1}{a^{2}}(l+(d-1) / 2)^{2}-((d-1) / 2-p)^{2}\left(\frac{1}{a^{2}}-1\right)
$$

which is not a perfect square unless $p=(d-1) / 2$. We therefore expect that a pole at $s=0$ in $\zeta_{p}^{\mathcal{M}}(s)$ will arise even for the case of the de Rham Laplacian. However, unlike the scalar $(p=0)$ case, it does not seem possible to achieve a perfect square, and hence to avoid the pole, simply by attaching a term to the de Rham Laplacian. This obstruction is probably related to the fact that there is no direct way of making the equation conformally invariant except for the special value $p=(d-1) / 2$. It is possible to use Branson's operator [9] but we would then lose contact with the body of work associated with the de Rham Laplacian.

The lack of conformal invariance can be appreciated in the structure of the coexact $\zeta$-function on the sphere, (44), which is a combination of improved 0 -form $\zeta$-functions each of which is conformal in a different dimension.

Further possible choices for $\mathcal{N}$ include hyperbolic spaces and factored spheres for which there would be more topological and analytical excitement involving, as they do, multiple connectedness and conical singularities.

Regarding spectral spinors, our previous method involved fitting a conjectured general form for the coefficients using specific numerical values. The present technique establishes both this general form and the actual expressions in one operation.

Acknowledgment. We wish to thank Peter Gilkey for help regarding the algebra behind reference [5], and thanks also to Michael Bordag for interesting discussions. KK was supported by the DFG under contract number Bo1112/4-1. 


\section{Appendix A.}

In this appendix we derive the form (44) for the coexact sphere $\zeta$ function. The most important property for the proof is the

\section{Lemma.}

$$
\begin{aligned}
\sum_{m=p+1}^{d}\left(\begin{array}{c}
m-1 \\
p
\end{array}\right) \zeta_{\mathcal{B}}\left(2 s ;(a+1) / 2 \mid \mathbf{1}_{m}\right) \\
=-\sum_{m=p}^{d-1}\left(\begin{array}{c}
m-1 \\
p-1
\end{array}\right) \zeta_{\mathcal{B}}\left(2 s ;(a-1) / 2 \mid \mathbf{1}_{m}\right) \\
+\left(\begin{array}{c}
d-1 \\
p
\end{array}\right) \zeta_{\mathcal{B}}\left(2 s ;(a-1) / 2 \mid \mathbf{1}_{d}\right)
\end{aligned}
$$

Proof. Rewrite the left-hand side of (97) using the difference relation, [1],

$$
\begin{aligned}
\zeta_{\mathcal{B}}\left(s ;(a+1) / 2 \mid \mathbf{1}_{m}\right)-\zeta_{\mathcal{B}}(s ;(a-1) / 2 \mid & \left.\mathbf{1}_{m}\right) \\
& =-\zeta_{\mathcal{B}}\left(s ;(a-1) / 2 \mid \mathbf{1}_{m-1}\right)
\end{aligned}
$$

and then combine terms using the binomial recursion (55).

Now we are set to show the following recursive property of the $p$-form $\zeta$-function (42)

\section{Lemma.}

$$
\begin{aligned}
\tilde{\zeta}_{p}^{S^{d}}(s)+\tilde{\zeta}_{p-1}^{S^{d-2}}(s)= & \left(\begin{array}{c}
d-1 \\
p
\end{array}\right) \tilde{\zeta}_{0}^{S^{d}}(s)+\delta_{p, 1}\left(\frac{d-3}{2}\right)^{-2 s} \\
& +\delta_{p, 0}\left(\frac{d-1}{2}\right)^{-2 s}
\end{aligned}
$$

for $0 \leq p \leq(d-1) / 2$. 
Proof. Starting from (42) and (15), use of (97) gives first

$$
\begin{aligned}
\tilde{\zeta}_{p}^{S^{d}}(s)= & -\sum_{m=p}^{d-1}\left(\begin{array}{c}
m-1 \\
p-1
\end{array}\right) \zeta_{\mathcal{B}}\left(2 s ;(d-1) / 2 \mid \mathbf{1}_{m}\right) \\
& +\left(\begin{array}{c}
d-1 \\
p
\end{array}\right) \zeta_{\mathcal{B}}\left(2 s ;(d-1) / 2 \mid \mathbf{1}_{d}\right) \\
& +\sum_{m=d-p}^{d}\left(\begin{array}{c}
m-1 \\
d-p-1
\end{array}\right) \zeta_{\mathcal{B}}\left(2 s ;(d+1) / 2 \mid \mathbf{1}_{m}\right)+\delta_{p, 0}\left(\frac{d-1}{2}\right)^{-2 s}
\end{aligned}
$$

Separate the $m=d$ term from the last sum, then

$$
\begin{aligned}
\tilde{\zeta}_{p}^{S^{d}}(s)= & \left(\begin{array}{c}
d-1 \\
p
\end{array}\right)\left(\zeta_{\mathcal{B}}\left(2 s ;(d+1) / 2 \mid \mathbf{1}_{d}\right)+\zeta_{\mathcal{B}}\left(2 s ;(d-1) / 2 \mid \mathbf{1}_{d}\right)\right) \\
& -\sum_{m=p}^{d-1}\left(\begin{array}{c}
m-1 \\
p-1
\end{array}\right) \zeta_{\mathcal{B}}\left(2 s ;(d-1) / 2 \mid \mathbf{1}_{m}\right)+\delta_{p, 0}\left(\frac{d-1}{2}\right)^{-2 s} \\
& +\sum_{m=d-p}^{d-1}\left(\begin{array}{c}
m-1 \\
d-p-1
\end{array}\right) \zeta_{\mathcal{B}}\left(2 s ;(d+1) / 2 \mid \mathbf{1}_{m}\right) .
\end{aligned}
$$

Next, combine the $m=d-1$ terms of both sums using (98),

$$
\begin{aligned}
\tilde{\zeta}_{p}^{S^{d}}(s)= & \left(\begin{array}{c}
d-1 \\
p
\end{array}\right) \tilde{\zeta}_{0}^{S^{d}}(s)-\sum_{m=p}^{d-2}\left(\begin{array}{c}
m-1 \\
p-1
\end{array}\right) \zeta_{\mathcal{B}}\left(2 s ;(d-1) / 2 \mid \mathbf{1}_{m}\right) \\
& +\sum_{m=d-p}^{d-2}\left(\begin{array}{c}
m-1 \\
d-p-1
\end{array}\right) \zeta_{\mathcal{B}}\left(2 s ;(d+1) / 2 \mid \mathbf{1}_{m}\right) \\
& -\left(\begin{array}{c}
d-2 \\
p-1
\end{array}\right) \zeta_{\mathcal{B}}\left(2 s ;(d-1) / 2 \mid \mathbf{1}_{d-2}\right)+\delta_{p, 0}\left(\frac{d-1}{2}\right)^{-2 s}
\end{aligned}
$$

Apply (97) once more in the second sum and then the binomial recursion, (55), to reach Lemma (99). Finally, applying this Lemma $p+1$ times, for $p<(d-1) / 2$, produces the important relation (44) in the main text. No doubt this also follows from a direct analysis of the degeneracies.

A comment on these equations is perhaps necessary. Setting $p=0$ in (99) we must recognise that $\tilde{\zeta}_{-1}^{S^{d-2}}(s)$ equals $((d-1) / 2)^{-2 s}$ for consistency. This follows easily from (42). In general, $\tilde{\zeta}_{-p}^{S^{d}}(s)=((d-1+2 p) / 2)^{-2 s}$. 


\section{Appendix B.}

In this appendix are listed some selected derivatives at zero of the coexact zeta function for absolute boundary conditions and with $\mathcal{M}$ the ball. In $d=2$,

$$
\begin{aligned}
& \zeta_{0}^{\prime \mathcal{M}}=-\frac{15}{32}-\frac{\log 2}{12}+\log 3-\frac{3 \zeta^{\prime}(-2)}{4}+\frac{5 \zeta^{\prime}(-1)}{2}+\zeta^{\prime}(0) \\
& \zeta_{1}^{\prime \mathcal{M}}=-\frac{1}{16}+\frac{11 \log 2}{6}-\frac{3 \zeta^{\prime}(-2)}{2}+3 \zeta^{\prime}(-1)-\zeta^{\prime}(0) \\
& \zeta_{2}^{\prime \mathcal{M}}=-\frac{3}{32}-\frac{\log 2}{12}-\frac{3 \zeta^{\prime}(-2)}{4}+\frac{\zeta^{\prime}(-1)}{2} .
\end{aligned}
$$

In $d=3$,

$$
\begin{aligned}
& \zeta_{0}^{\prime \mathcal{M}}=-\frac{1213}{4320}+\frac{151 \log 2}{90}+\frac{\zeta^{\prime}(-3)}{3}+\frac{\zeta^{\prime}(-2)}{2}+\frac{13 \zeta^{\prime}(-1)}{6}+\zeta^{\prime}(0) \\
& \zeta_{1}^{\prime \mathcal{M}}=\frac{5989}{10080}-\frac{19 \log 2}{30}+\zeta^{\prime}(-3)+\frac{\zeta^{\prime}(-2)}{2}-\frac{3 \zeta^{\prime}(-1)}{2}-\zeta^{\prime}(0) \\
& \zeta_{2}^{\prime \mathcal{M}}=-\frac{507}{1120}+\frac{7 \log 2}{10}+\zeta^{\prime}(-3)-\frac{\zeta^{\prime}(-2)}{2}-\frac{7 \zeta^{\prime}(-1)}{2}+2 \zeta^{\prime}(0) \\
& \zeta_{3}^{\prime \mathcal{M}}=\frac{173}{30240}+\frac{\log 2}{90}+\frac{\zeta^{\prime}(-3)}{3}-\frac{\zeta^{\prime}(-2)}{2}+\frac{\zeta^{\prime}(-1)}{6} .
\end{aligned}
$$

In $d=4$,

$$
\begin{aligned}
\zeta_{0}^{\prime \mathcal{M}}= & -\frac{25381}{46080}+\frac{17 \log 2}{2880}+\log 5-\frac{5 \zeta^{\prime}(-4)}{64}+\frac{23 \zeta^{\prime}(-3)}{48} \\
& +\frac{47 \zeta^{\prime}(-2)}{32}+\frac{103 \zeta^{\prime}(-1)}{48}+\zeta^{\prime}(0) \\
\zeta_{1}^{\prime \mathcal{M}}= & \frac{5803}{11520}+\frac{77 \log 2}{720}-\log 3-\frac{5 \zeta^{\prime}(-4)}{16}+\frac{19 \zeta^{\prime}(-3)}{12} \\
& +\frac{17 \zeta^{\prime}(-2)}{8}-\frac{25 \zeta^{\prime}(-1)}{12}-\zeta^{\prime}(0) \\
\zeta_{2}^{\prime \mathcal{M}}= & \frac{209}{2560}-\frac{863 \log 2}{480}-\frac{15 \zeta^{\prime}(-4)}{32}+\frac{15 \zeta^{\prime}(-3)}{8} \\
& -\frac{3 \zeta^{\prime}(-2)}{16}-\frac{21 \zeta^{\prime}(-1)}{8}+\zeta^{\prime}(0) \\
\zeta_{3}^{\prime \mathcal{M}}= & -\frac{2509}{11520}+\frac{77 \log 2}{720}-\frac{5 \zeta^{\prime}(-4)}{16}+\frac{11 \zeta^{\prime}(-3)}{12} \\
& -\frac{7 \zeta^{\prime}(-2)}{8}+\frac{19 \zeta^{\prime}(-1)}{12}-\zeta^{\prime}(0) \\
\zeta_{4}^{\mathcal{M}}= & \frac{47}{9216}+\frac{17 \log 2}{2880}-\frac{5 \zeta^{\prime}(-4)}{64}+\frac{7 \zeta^{\prime}(-3)}{48}-\frac{\zeta^{\prime}(-2)}{32}-\frac{\zeta^{\prime}(-1)}{48} .
\end{aligned}
$$


The $\zeta$-functions on the right are Riemann $\zeta$-functions.

As mentioned in the text, it is possible to obtain the determinants in any dimension $d$ and for any value of $p$ without difficulty.

\section{Appendix C.}

In this appendix we outline a method for the calculation of integrals of the type

$$
\int_{a}^{b} d w w^{n-1} \log \Gamma(w)
$$

needed in order to see explicitly the agreement of our results with those of [21].

We start from the usual relation

$$
\frac{\partial}{\partial w} \zeta(s, w)=-s \zeta(s+1, w)
$$

valid for any modified $\zeta$-function, such as $\zeta_{p}^{\mathcal{N}}(s)$ or the Barnes $\zeta$-function, but here applied to the Hurwitz $\zeta$-function.

The differentiations are continued to give,

$$
\begin{aligned}
\frac{\partial^{n}}{\partial w^{n}} \zeta(s, w) & =(-1)^{n} s(s+1) \ldots(s+n-1) \zeta(s+n, w) \\
& =f_{n}(s) \zeta(s+n, w) .
\end{aligned}
$$

Now differentiate with respect to $s$

$$
\frac{\partial^{n}}{\partial w^{n}} \zeta^{\prime}(s, w)=f_{n}^{\prime}(s) \zeta(s+n, w)+f_{n}(s) \zeta^{\prime}(s+n, w)
$$

and set $s=-n$

$$
\begin{aligned}
\frac{\partial^{n}}{\partial w^{n}} \zeta^{\prime}(-n, w) & =f_{n}^{\prime}(-n) \zeta(0, w)+f_{n}(-n) \zeta^{\prime}(0, w) \\
& =f_{n}^{\prime}(-n) \zeta(0, w)+f_{n}(-n)(\log \Gamma(w)-\log \sqrt{2 \pi})
\end{aligned}
$$

Next, multiply by $w^{n-1}$ and integrate

$$
\begin{aligned}
f_{n}(-n) \int_{a}^{b} d w w^{n-1} \log \Gamma(w)= & \int_{a}^{b} d w w^{n-1} \frac{\partial^{n}}{\partial w^{n}} \zeta^{\prime}(-n, w) \\
& +\frac{f_{n}(-n)}{n}\left(b^{n}-a^{n}\right) \log \sqrt{2 \pi} \\
& -f_{n}^{\prime}(-n) \int_{a}^{b} d w w^{n-1} \zeta(0, w)
\end{aligned}
$$


Define

$$
I_{n}(s)=\int_{a}^{b} d w w^{n-1} \frac{\partial^{n}}{\partial w^{n}} \zeta^{\prime}(s, w)
$$

and integrate by parts to get the recursion

$$
I_{n}(s)=\left.w^{n-1} \frac{\partial^{n-1}}{\partial w^{n-1}} \zeta^{\prime}(s, w)\right|_{a} ^{b}-(n-1) I_{n-1}(s)
$$

which is trivially continued using $I_{1}(s)=\zeta^{\prime}(s, b)-\zeta^{\prime}(s, a)$

From (103) we have

$$
\begin{aligned}
w^{m} \frac{\partial^{m}}{\partial w^{m}} \zeta^{\prime}(-n, w)= & w^{m}\left(f_{m}^{\prime}(-n) \zeta(-n+m, w)\right. \\
& \left.+f_{m}(-n) \zeta^{\prime}(-n+m, w)\right)
\end{aligned}
$$

where

$$
f_{m}(-n)=m !\left(\begin{array}{c}
n \\
m
\end{array}\right), \quad f_{m}^{\prime}(-n)=m !\left(\begin{array}{c}
n \\
m
\end{array}\right) \sum_{k=1}^{m} \frac{1}{m-n-k} .
$$

Using the recursion (106) $n$ times, and also (108), the following result is found for the integral (100),

$$
\begin{gathered}
\int_{a}^{b} d w w^{n-1} \log \Gamma(w)=\left\{\frac{1}{2 n}\left[\log (2 \pi)+\sum_{k=1}^{n} \frac{1}{k}\right] w^{n}-\frac{1}{n+1} w^{n+1} \sum_{k=1}^{n} \frac{1}{k}\right. \\
\left.+\sum_{l=1}^{n} \frac{(-1)^{l-1}}{l}\left(\begin{array}{c}
n-1 \\
l-1
\end{array}\right) w^{n-l}\left[\zeta^{\prime}(-l, w)-\zeta(-l, w) \sum_{k=1}^{n-l} \frac{1}{k+l}\right]\right\}\left.\right|_{a} ^{b}
\end{gathered}
$$

The simplest case, when $n=1$, is given in [20] and an integral related to (109) for $n=2$ can be found in Nash and O'Connor, [33] eqn.(C.30).

If one of the limits in (100) is zero, the last sum in (109) has to be interpreted as the limit as $w \rightarrow 0$ and, in this case, we use

$$
\zeta(s, w)=w^{-s}+\zeta(s, 1+w)
$$

to see that

$$
\lim _{w \rightarrow 0} w^{n-l}\left[\zeta^{\prime}(-l, w)-\zeta(-l, w) \sum_{k=1}^{n-l} \frac{1}{k+l}\right]=0
$$


for $l<n$, whereas

$$
\lim _{w \rightarrow 0} \zeta^{\prime}(-n, w)=\zeta^{\prime}(-n)
$$

for $n \geq 1$.

Let us now give some examples of the general formula (109). To compare with $\zeta_{0}^{\prime}(0)$ in $d=3$ of [21], we need

$$
\int_{0}^{1} d w(w-1) \log \Gamma(w)
$$

which is obtained by setting $n=1$ and $n=2$. For $n=1$, we get the well known formula ([23], 1.9.1 (18))

$$
\int_{0}^{1} d w \log \Gamma(w)=\frac{1}{2} \log (2 \pi)=-\zeta^{\prime}(0)
$$

which is the essential part of Raabe's formula.

For $n=2$

$$
\int_{0}^{1} d w w \log \Gamma(w)=-\frac{1}{12}-\frac{1}{2} \zeta^{\prime}(0)+\zeta^{\prime}(-1) .
$$

The integral term in [21] is therefore

$$
2 \int_{0}^{1} d w(w-1) \log \Gamma(w)=-\frac{1}{6}+2 \zeta^{\prime}(-1)+\zeta^{\prime}(0) .
$$

In other dimensions, generally $a$ and $b$ take integer or half-integer values. Using (109) the integrals can always be expressed in terms of derivatives of the Riemann $\zeta$-function. We meet some known integrals such as $([37,20])$

$$
\int_{1}^{3 / 2} d w \log \Gamma(w)=-\frac{3}{8}-\frac{13}{24} \log 2-\frac{3}{2} \zeta^{\prime}(-1)-\frac{1}{2} \zeta^{\prime}(0)
$$

and some lesser known ones, e.g.

$$
\int_{0}^{3 / 2} d w w \log \Gamma(w)=-\frac{1}{2}-\frac{11}{16} \log 2+\frac{7}{8} \zeta^{\prime}(-2)-\frac{3}{4} \zeta^{\prime}(-1)-\frac{9}{8} \zeta^{\prime}(0)
$$

generalisations of which can be found using (109).

Finally we point out that a generalisation of Raabe's formula to the multiple $\Gamma$-function is derived by Barnes [1] using the same ingredients as in the above. 


\section{Appendix D.}

The identity (86) will now be proved. In terms of the spinor base $\zeta-$ function it reads

$$
\operatorname{Res} \zeta_{\mathcal{N}}(1 / 2)-\operatorname{Res} \zeta_{\mathcal{N}}(1)-2 \sum_{i=1}^{d-2} \operatorname{Res} \zeta_{\mathcal{N}}(1+i / 2) D_{i}^{\prime}(1)=0
$$

Using knowledge of the residues in terms of the Bernoulli functions, [1], one has

$$
\begin{aligned}
0=\frac{B_{d-1}^{(d)}((d-1) / 2)}{(d-1) !} & +\frac{B_{d-2}^{(d)}((d-1) / 2)}{(d-2) !} \\
& +2 \sum_{i=1}^{d-2}(-1)^{i} D_{i}^{\prime}(1) \frac{B_{d-2-i}^{(d)}((d-1) / 2)}{(1+i) !(d-2-i) !}
\end{aligned}
$$

which is a standard recursion formula for the generalized Bernoulli functions as will now be shown.

We first note that $D_{i}^{\prime}(1)=(-1)^{i} / 2$. This value is obtained by comparing the small $z$ approximation of the Bessel function $I_{\nu}(\nu z)$ with Olver's asymptotic form. Thus, working always to order $z^{2}$,

$$
\ln I_{\nu}(\nu z) \sim \nu \ln (\nu z / 2)-\ln \Gamma(1+\nu)+\ln \left(1+\frac{1}{4(\nu+1)}(\nu z)^{2}\right)
$$

Expanding the log,

$$
\ln \left(1+\frac{1}{4(\nu+1)}(\nu z)^{2}\right) \sim \frac{1}{4} z^{2} \frac{\nu}{1+1 / \nu}=\frac{1}{4} z^{2} \nu \sum_{n=0}^{\infty}(-1)^{n} \nu^{-n}
$$

and also

$$
\ln \Gamma(1+\nu) \sim \nu \ln \nu-\nu+\frac{1}{2} \ln 2 \pi \nu-\sum_{k=1}^{\infty} \frac{\zeta_{R}(-k)}{k} \nu^{-k}
$$


The Bessel asymptotics are

$$
\begin{aligned}
\ln I_{\nu}(\nu z) \sim- & \frac{1}{2} \ln 2 \pi \nu-\frac{1}{4} \ln \left(1+z^{2}\right) \\
& +\nu\left(\sqrt{1+z^{2}}+\ln \left(\frac{z}{1+\sqrt{1+z^{2}}}\right)\right)+\sum_{n=1}^{\infty} \frac{D_{n}(t)}{\nu^{n}} \\
\sim- & \frac{1}{2} \ln 2 \pi \nu-\frac{1}{4} z^{2} \\
& +\nu\left(1+\frac{1}{4} z^{2}+\ln (z / 2)\right)+\sum_{n=1}^{\infty} \frac{D_{n}(1)-z^{2} D_{n}^{\prime}(1) / 2}{\nu^{n}} .
\end{aligned}
$$

Comparing, we obtain the quoted value of $D_{n}^{\prime}(1)$, and also that of $D_{n}(1)$ met in our previous work. The higher derivatives can clearly be found by carrying the expansion further.

Substituting these values of $D_{n}^{\prime}(1)$ into (115) yields the condition

$$
\left.\sum_{j=0}^{d-1}\left(\begin{array}{c}
d-1 \\
j
\end{array}\right) B_{d-1-j}^{(d)}((d-1) / 2)\right)=0
$$

which is a special case of the recursion relation ([34] eqn.(11) p.161)

$$
\sum_{j=0}^{\nu}\left(\begin{array}{l}
\nu \\
j
\end{array}\right) \omega^{j} B_{\nu-j}^{(n)}(x \mid \omega)=\omega \nu B_{\nu-1}^{(n-1)}\left(x \mid \omega^{\prime}\right), \quad \omega=\left(\omega^{\prime}, \omega\right)
$$

involving the general Bernoulli function. Setting $\omega=1, \nu=d-1$ and $n=d$, this becomes

$$
\begin{aligned}
\sum_{j=0}^{d-1}\left(\begin{array}{c}
d-1 \\
j
\end{array}\right) B_{d-1-j}^{(d)}(x) & =(d-1) B_{d-2}^{(d-1)}(x) \\
& =(d-1)(x-1)(x-2) \ldots(x-d+2) .
\end{aligned}
$$

If $d$ is odd, $(d-1) / 2$ is integral and, since $1<(d-1) / 2 \leq d-2$ for $d>1$, the right-hand side of (118) vanishes for all odd $d$ when $x=(d-1) / 2$, as required to show (116).

For even $d=2 q,(118)$ allows one to give the general form of the coefficient

$$
A_{(D-2) / 2}^{(S)}(D)=(-1)^{q}\left(q-\frac{1}{2}\right)\left(\left(q-\frac{3}{2}\right) \ldots \frac{3}{2}\right)^{2}, \quad D=2 q+1 .
$$


A similar result should hold for the monopole. Put $\omega=a$ in (117).

\section{References.}

[1] E.W. Barnes, On the Theory of the multiple Gamma function, Trans. Camb. Phil. Soc. 19 (1903), 374.

[2] E.W. Barnes, On the asymptotic expansion of integral functions of multiple linear sequence, Trans. Camb. Phil. Soc. 19 (1903), 426.

[3] A.O. Barvinsky, Yu.A. Kamenshchik and I.P. Karmazin, One-loop quantum cosmology, Ann. Phys. 219 (1992), 201.

[4] B.L. Beers and R.S. Millman, The spectra of the Laplace-Beltrami operator on compact, semisimple Lie groups, Am. J. Math. 99 (1975), 801-807.

[5] N. Blažić, N. Bokan P.B. and Gilkey, Spectral geometry of the form valued Laplacian for manifolds with boundary, Ind. J. Pure and Appl. Math. 23 (1992), 103-120.

[6] M. Bordag, E. Elizalde and K. Kirsten, Heat kernel coefficients of the Laplace operator on the D-dimensional ball, J. Math. Phys. 37 (1996), 895.

[7] M. Bordag, B. Geyer, K. Kirsten and E. Elizalde, Zeta function determinant of the Laplace operator on the D-dimensional ball, Comm. Math. Phys. 179 (1996), 215.

[8] M. Bordag, K.Kirsten and J.S. Dowker, Heat kernels and functional determinants on the generalized cone, Comm. Math. Phys. 182 (1996), 371.

[9] T.P. Branson, Conformally covariant equations on differential forms, Comm. Partial Diff. Equns. 7 (1982), 393-431.

[10] A.A. Bytsenko, G. Cognola and S. Zerbini, Quantum fields in hyperbolic spacetimes with finite spatial volume, Class. Quant. Grav. 14 (1997), 615.

[11] A.A. Bytsenko, G. Cognola and S. Zerbini, Determinant of Laplacian on a non-compact 3-dimensional hyperbolic manifold with finite volume, J. Phys. A30 (1997), 3543.

[12] R. Camporesi and A. Higuchi, Plancherel measure for $p$-forms in real hyperbolic space, J. Geom. and Phys. 15 (1994), 57-94.

[13] Peter Chang and J.S.Dowker, Vacuum energy on orbifold factors of spheres, Nucl. Phys. B395 (1993), 407.

[14] J. Cheeger, Spectral Geometry of Singular Riemannian Spaces, J. Diff. Geom. 18 (1983), 575. 
[15] J. Cheeger, Hodge theory of complex cones, Astérisque 101-102 (1983), 118134.

[16] J. Cheeger, Analytic torsion and the heat equation, Ann. of Math. 109 (1979), 259-322.

[17] E. Copeland and D.J. Toms, Quantized antisymmetric tensor fields and self-consistent dimensional reduction in higher-dimensional spacetimes, Nucl. Phys. B255 (1985), 201.

[18] J.S. Dowker, Effective action on spherical domains, Comm. Math. Phys. 162 (1994), 633.

[19] J.S. Dowker, J.S. Apps, M. Bordag and K. Kirsten, Spectral invariants for the Dirac equation with various boundary conditions, Class. Quant. Grav. 13 (1996), 2911.

[20] E. Elizalde and A. Romeo, An integral involving the generalized zeta function, International J. of Math. and Phys. 13 (1994), 453.

[21] E. Elizalde, M. Lygren and D.V. Vassilevich, Zeta function for the laplace operator acting on forms in a ball with gauge boundary conditions, Comm. Math. Phys. 183 (1997), 645.

[22] E. Elizalde, M. Lygren and D.V. Vassilevich, Antisymmetric tensor fields on spheres: functional determinants and non-local counterterms, J. Math. Phys. 37 (1996), 3105.

[23] A. Erdelyi, W. Magnus, F. Oberhettinger and F.G.Tricomi, Higher Transcendental Functions, Vol.I, McGraw-Hill, New York, 1953.

[24] G. Esposito,Quantum Gravity, Quantum Cosmology and Lorentzian Geometries, Lecture Notes in Physics, Monographs, Vol. m12, Springer-Verlag, Berlin 1994.

[25] S. Gallot and D. Meyer, Opérateur de coubure et Laplacian des formes différentielles d'une variété riemannienne, J. Math. Pures. Appl. 54 (1975), 289.

[26] P.B. Gilkey, Invariance theory, the heat equation and the Atiyah-Singer index theorem, 2nd. Edn., CTC Press, Boca Raton 1995.

[27] P. Günther and R. Schimming, Curvature and spectrum of compact Riemannian manifolds, J. Diff. Geom. 12 (1977), 599-618.

[28] A. Ikeda and Y. Taniguchi, Spectra and eigenforms of the Laplacian on $S^{n}$ and $P^{n}(C)$, Osaka J.Math. 15 (1978), 515-546. 
[29] I. Iwasaki and K. Katase, On the spectra of Laplace operator on $\Lambda^{*}\left(S^{n}\right)$, Proc. Jap. Acad. A55 (1979), 141.

[30] K. Kirsten and G. Cognola, Heat-kernel coefficients and functional determinants for higher spin fields on the ball, Class. Quant. Grav. 13 (1996), 633-644.

[31] M. Levitin, Dirichlet and Neumann invariants for Euclidean balls, Diff. Geom. and its Appl., 8 (1998), 35.

[32] I.G. Moss and S.J. Poletti, Conformal anomaly on an Einstein space with boundary, Phys. Lett. B333 (1994), 326.

[33] C. Nash and D.J. O'Connor, Determinants of Laplacians, the Ray-Singer torsion on lens spaces and the Riemann zeta function, J. Math. Phys. 36 (1995), 1462.

[34] N.E. Nörlund, Mémoire sur les polynomes de Bernoulli, Acta Mathematica 4 (1921), 1922.

[35] D.B. Ray, Reidemeister torsion and the Laplacian on lens spaces, Adv. in Math. 4 (1970), 109.

[36] I.H. Russell and D.J. Toms, Vacuum energy for massive forms in $R^{m} \times S^{N}$, Class. Quant. Grav. 4 (1986), 1357.

[37] A. Voros, Spectral functions, special functions and the Selberg zeta function, Comm. Math. Phys. 110 (1987), 439.

Department of Theoretical Physics, The University of MANChester

MANCHESTER, ENGLAND

AND

UNIVERSITÄT LEIPZIG

InSTITUT FÜR TheORETISChe PHYSIK

Augustusplatz 10, 04109

LeIPZIG, Germany

E-mail addresses: dowker@a3.ph.man.ac.uk

Klaus.Kirsten@itp.uni-leipzig.de 